\title{
Flows driven by rough paths
}

\author{
Ismaël Bailleul
}

\begin{abstract}
We devise in this work a simple mechanism for constructing flows on a Banach space from approximate flows, and show how it can be used in a simple way to reprove from scratch and extend the main existence and well-posedness results for rough differential equations, in the context of dynamics on a Banach space driven by a Hölder weak geometric rough path; the explosion question under linear growth conditions, Taylor expansion and Euler estimates are also dealt with. We illustrate our approach by proving an existence and well-posedness result for some mean field stochastic rough differential equation.
\end{abstract}

\section{Introduction}

Since T. Lyons wrote his groundbreaking article [19] on rough paths, there has been a constantly growing industry in understanding the scope of the theory. Besides providing an alternative enlighting view on Itô's theory of stochastic integration and some deep associated results, rough paths theory has now invaded the world of numerical simulations, stochastic and deterministic partial differential equations and finance, to name but a few areas. Despite Lyons' Saint Flour lecture notes [20], his book [21] with Qian and the impressive and exhaustive book [16] of Friz and Victoir, rough path still seems to be seen as a somewhat difficult and technical subject where algebra and classical analysis meet in an intricate way.

We show in this work how the main existence and uniqueness results of the theory can be proved from scratch using a simple mechanism for constructing flows from approximate flows, in the context of dynamics in a Banach space driven by Hölder weak geometric rough paths. Contrary to Lyons, Friz-Victoir or Gubinelli's approach, we work primarily with maps from the state space $E$ to itself rather than with $E$-valued paths. Our dynamics on the space of maps $\varphi$ will have typical form

$$
d \varphi=V d t+\mathrm{F} \mathbf{X}(d t),
$$

Mathematics Subject Classification (2010): 34A12, 34A45, 34A99.

Keywords: Rough differential equations, flows, approximate flows, mean field differential equation. 
for some driving vector fields $V$ and $\mathrm{F}=\left(V_{1}, \ldots, V_{\ell}\right)$ and a (Hölder weak geometric $p$-) rough path $\mathbf{X}$ on some time interval $[0, T]$. Roughly speaking, a rough path consists of an $\mathbb{R}^{\ell}$-valued non-smooth path $X$ together with a number of objects which play the role of the missing iterated integrals of $X$, in the sense that they satisfy the same algebraic relations as the iterated integrals of any smooth path, as well as some natural size requirements. Given such a rough path and some vector fields $V, V_{1}, \ldots, V_{\ell}$, a family $\left(\mu_{t s}\right)_{0 \leqslant s \leqslant t \leqslant T}$ of diffeomorphisms of $E$ is constructed from an ordinary differential equation. These maps do not form a flow, in the sense that $\mu_{t s}$ is not equal to $\mu_{t u} \circ \mu_{u s}$ for all $0 \leqslant s \leqslant u \leqslant t \leqslant T$. However, some conditions on the driving vector fields and the rough path ensure the existence of a unique flow of maps $\left(\varphi_{t s}\right)_{0 \leqslant s \leqslant t \leqslant T}$ close to $\left(\mu_{t s}\right)_{0 \leqslant s \leqslant t \leqslant T}$. This is what we call in that case the solution to the rough differential equation (1.1) on flows.

All other approaches to rough paths consider paths associated with a point motion as the fundamental basic object. Lyons, and later Gubinelli, interpret (1.1) as an integral equation, which requires a suitable notion of rough path integral as a mechanism to attach to a rough path $\mathbf{Y}$ and some sufficiently regular one form $g$ another rough path $\int_{0}^{*} g(\mathbf{Y}) d \mathbf{Y}$. Solving equation (1.1) then amounts to find a fixed point to an integral equation of the form $\mathbf{Y}=\int_{0}^{\cdot} g(\mathbf{Y}) d \mathbf{Y}$, where $\mathbf{Y}$ is some extension of the original rough path $\mathbf{X}$. See for instance [19], [20] or [21], and Friz and Hairer's review of Gubinelli's approach [12]. Davie's definition takes as a starting point the fact that a solution of a controlled ordinary differential equation $d x_{t}=\sum_{i=1}^{\ell} V_{i}\left(x_{t}\right) d h_{t}^{i}$, with $h$ smooth, admits an Euler expansion

$$
x_{t}-x_{s}=\sum_{i=1}^{\ell}\left(h_{t}-h_{s}\right)^{i} V_{i}(x-s)+\sum_{j, k=1}^{\ell}\left(\int_{s}^{t}\left(h_{r}-h_{s}\right)^{k} d h_{r}^{j}\right)\left(V_{j} V_{k}\right)\left(x_{s}\right)+o\left(|t-s|^{2}\right)
$$

which describes the path $x_{\bullet}$ accurately enough to characterize it uniquely. A $p$ rough path $\mathbf{X}=\left(X^{1}, X^{2}\right)$, with $2 \leqslant p<3$, provides quantities $X_{t s}^{1} \in \mathbb{R}^{\ell}$ and $X_{t s}^{2} \in M_{\ell}(\mathbb{R})$, for $0 \leqslant s \leqslant t \leqslant T$, which, when plugged into formula (1.2) in place of $h_{t}-h_{s}$ and $\int_{s}^{t}(\cdots)$, with a different $o(\cdot)$ term, describe accurately a unique $\mathbb{R}^{d}$ valued path. Friz and Victoir extended Davie's picture to any geometric Hölder $p$ rough path by interpreting Davie's picture as a way of constructing $\mathbb{R}^{d}$-valued paths as limits in some appropriate topology of paths generated by controlled ordinary differential equations in which the control converges in a rough path sense to some limit rough path. With such a view, no notion of integral is needed to define a dynamic. Neither is it the case in the approach developed in this work, whose core is a simple and non-commutative extension of Feyel-de la Pradelle' sewing lemma [10], [11], well-adapted to the construction of flows of maps, and totally independent of any problem about rough paths; it is the subject of section 2 .

It comes as a nice feature of our approach that convergence estimates for Euler/Milstein type schemes and Taylor expansion for solution flows come almost for free; this is explained in section 3.3. The approach is sufficiently robust to work with vector fields with at most linear growth and prove non-explosion of the dynamics in that case, as explained in section 4 . The results of section 3 on flows driven by rough paths are applied in section 5 to give a simple proof of the main 
existence/uniqueness results for the classical rough differential equations on paths driven by Hölder weak geometric. They are illustrated differently in section 6 where we prove a well-posedness result for some large class of mean field stochastic rough differential equation.

Although it is true that the classical approach to rough paths eventually leads with some work to the construction of solution flows to equation (1.1) in our sense, this work points out the fact that it is fruitful to take the converse direction and consider flows as basic objects rather than paths. This change of point of view has several advantages over the usual approach, the first of which being that we are able to give a simple toolkit for constructing flows in a Banach space, which provides an almost technical-free approach to rough differential equations. Our main results on flows driven by rough paths,

- well-posedness of the rough differential equation (1.1) on flows, giving back and extending sharp existence and uniqueness results for classical rough differential equations,

- non-explosion for dynamics driven by weak geometric rough paths under linear growth conditions on the vector fields,

- existence and well-posedness results for some mean field rough differential equations,

hold for any weak geometric Hölder $p$-rough path. We have chosen to give the proof of these facts in the setting of a finite dimensional driving signal, to keep the exposition at an elementary level. The extension of our results to Banach spacevalued rough paths is done in [2], giving back Lyons' theory in its full force. In another direction, the machinery of $\mathcal{C}^{1}$-approximate flows introduced in section 2 , is used in the forthcoming work [3] to recover and extend the theory of stochastic flows developed in the 80's and 90's by Le Jan, Watanabe, Kunita and others.

A few notations will be used throughout the text, which we gather here.

- $(E,|\cdot|)$ will denote Banach space and $\mathrm{L}(E)$ the set of linear continuous maps from $E$ to itself.

- Fix $T>0$ and a non-integer $\gamma>1$, with integer part $[\gamma]$ and fractional part $\{\gamma\}$. We say that a function or a vector field $W$ on $E$ is $\gamma$-Lipschitz if it is $\mathcal{C}^{[\gamma]}$ with a $\{\gamma\}$-Hölder continuous differential of order $[\gamma]$, and $W$ and all its derivative are bounded. Its $\gamma$-Lipschitz norm $\|W\|_{\gamma}$ is defined as

$$
\|W\|_{\gamma}=\sum_{r=0}^{[\gamma]}\left|W^{(r)}\right|+\left\|W^{([\gamma])}\right\|_{\{\gamma\}}<\infty,
$$

where $W^{(r)}$ is the differential of order $r$ of $W$, with operator norm $\left|W^{(r)}\right|$, and $\left\|W^{([\gamma])}\right\|_{\{\gamma\}}$ stands for the classical $\{\gamma\}$-Hölder norm of $W^{([\gamma])}$.

- Given some sufficiently regular vector fields $V_{1}, \ldots, V_{\ell}$ on $E$ and a tuple $I=\left(i_{1}, \ldots, i_{r}\right) \in \llbracket 1, \ell \rrbracket^{r}$, we identify vector fields with derivation operators and write $V_{I}$ for the differential operator

$$
f \in \mathcal{C}^{r} \mapsto V_{i_{1}}\left(V_{i_{2}}\left(\ldots V_{i_{r}} f\right)\right) \in \mathcal{C}^{0} .
$$


Writing $[V, W]$ for the bracket of two vector fields, this defines a first order differential operator, that is a vector field. Set

$$
V_{[I]}=\left[V_{i_{1}},\left[V_{i_{2}}, \ldots,\left[V_{i_{r-1}}, V_{i_{r}}\right]\right] \ldots\right] .
$$

- We identify in the sequel $L\left(\mathbb{R}^{\ell}\right)=\mathbb{R}^{\ell} \otimes\left(\mathbb{R}^{\ell}\right)^{*}$ and $\mathbb{R}^{\ell} \otimes \mathbb{R}^{\ell}$ with $M_{\ell}(\mathbb{R})$, via the matrix representation of linear maps in the canonical basis. In these terms, given any two vectors $x, y$ of $\mathbb{R}^{\ell}$, the $(j k)$-th component $(x \otimes y)^{j k}$ of $x \otimes y$ is $x^{j} y^{k}$.

- We use the convention that $a^{i} b_{i}$ stands for the sum $\sum_{i} a^{i} b_{i}$.

- Constants depending only on the appropriate norms of some given vector fields are said to depend on the data of the problem.

- We use the letter $c$ for constants depending on the data of the problem, and whose value may change from place to place.

- The notation $O_{c}(A)$ stands for a quantity whose norm or absolute value is bounded above by $c A$.

\section{Constructing flows on a Banach space}

Let $E$ be a Banach space. Recall that a flow on $E$ is a family $\left(\varphi_{t s}\right)_{0 \leqslant s \leqslant t \leqslant T}$ of maps from $E$ to itself such that $\varphi_{t s}=\varphi_{t u} \circ \varphi_{u s}$, for all $0 \leqslant s \leqslant u \leqslant t \leqslant T$, and $\varphi_{s s}=\mathrm{Id}$, for all $0 \leqslant s \leqslant T$. We provide in this section a simple tool for constructing flows on $E$, which rests on an elementary extension of Feyel-de la Pradelle sewing lemma [10] to the non-commutative setting of maps from $E$ to itself. Given a family of maps $\left(\mu_{t s}\right)_{0 \leqslant s \leqslant t \leqslant T}$, set

$$
\mu_{t s}^{n}=\bigcirc_{i=0}^{n-1} \mu_{s_{i+1} s_{i}},
$$

with $s_{i}=s+\frac{i}{n}(t-s)$. Given a partition $\pi_{t s}=\left\{s=s_{0}<s_{1}<\cdots<s_{n-1}<s_{n}=t\right\}$ of $(s, t)$, set

$$
\mu_{\pi_{t s}}=\mu_{s_{n} s_{n-1}} \circ \cdots \circ \mu_{s_{1} s_{0}} .
$$

Theorem 2.1 (Sewing lemma for flows). Let $\left(\mu_{t s}\right)_{0 \leqslant s \leqslant t \leqslant T}$ be a family of $\mathcal{C}^{1}$ maps from $E$ to itself, depending continuously on $(s, t)$ in the uniform topology, and enjoying the following two properties.

H1. (Regularity). There exists two positive constants $\alpha$ and $\rho$, with

$$
0<1-\rho<\alpha<1,
$$

such that the maps $\mu_{t s}$ are $(1+\rho)$-Lipschitz, and one has

$$
D_{x} \mu_{t s}=\mathrm{Id}+A_{x}^{t s}+B_{x}^{t s},
$$

for all $x \in E$, for some $\mathrm{L}(E)$-valued $\rho$-Lipschitz maps $A^{\text {ts }}$ on $E$, with $\rho$ Lipschitz norm bounded above by $c|t-s|^{\alpha}$, and some $\mathrm{L}(E)$-valued $\mathcal{C}^{1}$ bounded maps $B^{\text {ts }}$ on $E$, with $\mathcal{C}^{1}$-norm bounded above by $o_{t-s}(1)$. 
H2. ( $\mathcal{C}^{1}$-approximate flow property). There exists a positive constant $c_{1}$ and $a>1$, such that one has

$$
\left\|\mu_{t u} \circ \mu_{u s}-\mu_{t s}\right\|_{\mathcal{C}^{1}} \leqslant c_{1}|t-s|^{a}
$$

for all $0 \leqslant s \leqslant u \leqslant t \leqslant T$.

Under these conditions, there exists a positive constant $\delta$ and a unique flow of maps $\left(\varphi_{t s}\right)_{0 \leqslant s \leqslant t \leqslant T}$ on E such that

$$
\left\|\varphi_{t s}-\mu_{t s}\right\|_{\infty} \leqslant c|t-s|^{a}
$$

holds for some positive constant $c$ and all $0 \leqslant s \leqslant u \leqslant t \leqslant T$, with $t-s \leqslant \delta$; furthermore, we have

$$
\left\|\varphi_{t s}-\mu_{\pi_{t s}}\right\|_{\infty} \leqslant 2 c_{1} T\left|\pi_{t s}\right|^{a-1}
$$

for any partition $\pi_{t s}$ of any interval $(s, t) \subset[0, T]$, of mesh $\left|\pi_{t s}\right| \leqslant \delta$.

A family of maps enjoying the properties listed in the above theorem is called a $\mathcal{C}^{1}$-approximate flow. A slightly weaker notion will also be useful. A family $\left(\mu_{t s}\right)_{0 \leqslant s \leqslant t \leqslant T}$ of Lipschitz continuous maps from $E$ to itself, with Lipschitz constant uniformly bounded above by $\left(1+o_{m}(1)\right)$, for any $0 \leqslant s \leqslant t \leqslant T$, with $t-s \leqslant m$ and any $m>0$, and such that

$$
\left\|\mu_{t u} \circ \mu_{u s}-\mu_{t s}\right\|_{\infty} \leqslant c_{1}^{\prime}|t-s|^{a}
$$

holds for for all $0 \leqslant s \leqslant u \leqslant t \leqslant T$, for some positive constant $c_{1}^{\prime}$, is called a $\mathcal{C}^{0}$-approximate flow.

To prepare the proof of Theorem 2.1, note that it is elementary to show that $\mathcal{C}^{0}$-approximate flows satisfy an a priori stronger form of identity than (2.5), as expressed in the next lemma, for which we introduce the following definition.

Definition 2.2. Let $\epsilon \in(0,1)$ be given. A partition $\pi=\left\{s=s_{0}<s_{1}<\cdots<\right.$ $\left.s_{n-1}<s_{n}=t\right\}$ of $(s, t)$ is said to be $\epsilon$-special if it is either trivial or

- one can find an $s_{i} \in \pi$ such that $\epsilon \leqslant \frac{s_{i}-s}{t-s} \leqslant 1-\epsilon$,

- and for any choice $u$ of such an $s_{i}$, the partitions of $[s, u]$ and $[u, t]$ induced by $\pi$ are both $\epsilon$-special.

A partition of any interval into subintervals of equal length is $\frac{1}{2}$-special. Given an $\epsilon$-special partition $\pi=\left\{s=s_{0}<s_{1}<\cdots<s_{n-1}<s_{n}=t\right\}$ of $(s, t)$ and $u \in\left\{s_{1}, \ldots, s_{n-1}\right\}$ with $\epsilon \leqslant \frac{u-s}{t-s} \leqslant 1-\epsilon$, the induced partitions of the intervals $[s, u]$ and $[u, t]$ are also $\epsilon$-special. Set

$$
m_{\epsilon}=\sup _{\epsilon \leqslant \beta \leqslant 1-\epsilon}\left\{\beta^{a}+(1-\beta)^{a}\right\}<1,
$$

and pick a constant

$$
L>\frac{2 c_{1}}{1-m_{\epsilon}}
$$

where $c_{1}$ is the constant that appears in the definition of a $\mathcal{C}^{1}$-approximate flow, in equation (2.2). 
Lemma 2.3. Let $\left(\mu_{t s}\right)_{0 \leqslant s \leqslant t \leqslant T}$ be a $\mathcal{C}^{0}$-approximate flow on $E$. Given $\epsilon>0$, there exists a positive constant $\delta$ such that for any $0 \leqslant s \leqslant t \leqslant T$ with $t-s \leqslant \delta$, and any special partition $\pi_{t s}$ of type $\epsilon$ of an interval $(s, t) \subset[0, T]$, we have

$$
\left\|\mu_{\pi_{t s}}-\mu_{t s}\right\|_{\infty} \leqslant L|t-s|^{a} .
$$

Proof. We proceed by induction on the number $n$ of subintervals of the partition. The case $n=2$ is identity (2.2). Suppose the statement has been proved for $n \geqslant 2$. Fix $0 \leqslant s<t \leqslant T$ with $t-s \leqslant \delta$, and let $\pi_{t s}=\left\{s_{0}=s<s_{1}<\cdots<s_{n}<\right.$ $\left.s_{n+1}=t\right\}$ be a partition of $(s, t)$ of special type $\epsilon$, splitting the interval $(s, t)$ into $(n+1)$ subintervals. Set $m=[(n+1) / 2]$ and $u=s_{m}$, so the two partitions $\pi_{t u}$ and $\pi_{u s}$ are both of special type $\epsilon$, with respective cardinals no greater than $n$, and $\epsilon \leqslant \frac{t-u}{t-s} \leqslant 1-\epsilon$. Then

$$
\begin{gathered}
\left\|\mu_{\pi_{t s}}-\mu_{t s}\right\|_{\infty} \leqslant\left\|\mu_{\pi_{t u}} \circ \mu_{\pi_{u s}}-\mu_{t u} \circ \mu_{\pi_{u s}}\right\|_{\infty}+\left\|\mu_{t u} \circ \mu_{\pi_{u s}}-\mu_{t s}\right\|_{\infty} \\
\leqslant\left\|\mu_{\pi_{t u}}-\mu_{t u}\right\|_{\infty}+\left\|\mu_{t u} \circ \mu_{\pi_{u s}}-\mu_{t u} \circ \mu_{u s}\right\|_{\infty} \\
\quad+\left\|\mu_{t u} \circ \mu_{u s}-\mu_{t s}\right\|_{\infty} \\
\leqslant L|t-u|^{a}+\left(1+o_{\delta}(1)\right) L|u-s|^{a}+c_{1}|t-s|^{a},
\end{gathered}
$$

by the induction hypothesis and the conditions defining a $\mathcal{C}^{0}$-approximate flow. Set $u-s=\beta(t-s)$, with $\epsilon \leqslant \beta \leqslant 1-\epsilon$. The above inequality rewrites

$$
\left\|\mu_{\pi_{t s}}-\mu_{t s}\right\|_{\infty} \leqslant\left\{\left(1+o_{\delta}(1)\right)\left((1-\beta)^{a}+\beta^{a}\right) L+c_{1}\right\}|t-s|^{a} .
$$

One closes the induction by choosing $\delta$ small enough to have $\left(1+o_{\delta}(1)\right) m_{\epsilon} \leqslant \frac{1+m_{\epsilon}}{2}$, for which choice the above term $\{\cdots\}$ is no greater than $L$.

Working with $\mathcal{C}^{1}$-approximate flows, one can strengthen this result to get a $\mathcal{C}^{1}$ estimate in equation (2.6) instead of a $\mathcal{C}^{0}$ estimate.

Lemma 2.4. Let now $\left(\mu_{t s}\right)_{0 \leqslant s \leqslant t \leqslant T}$ be a $\mathcal{C}^{1}$-approximate flow on $E$. Given $\epsilon>0$, there exists a positive constant $\delta$ such that

$$
\left\|\mu_{\pi_{t s}}-\mu_{t s}\right\|_{\mathcal{C}^{1}} \leqslant L|t-s|^{a}
$$

for any partition $\pi_{t s}$ of $(s, t)$ of special type $\epsilon$, whenever $t-s \leqslant \delta$.

Proof. Given the preceding result, we need to prove that we have

$$
\left|D_{x}\left(\mu_{\pi_{t s}}\right)-D_{x} \mu_{t s}\right| \leqslant L|t-s|^{a}
$$

for all $x \in E$, for an appropriate choice of $\delta$. We proceed by induction on the number $n$ of subintervals of the partition as in the proof of Lemma 2.3.

The case $n=2$ is identity (2.2). Suppose the statement has been proved for $n \geqslant 2$. Fix $0 \leqslant s<t \leqslant T$ with $t-s \leqslant \delta$, and let $\pi_{t s}=\left\{s_{0}=s<s_{1}<\cdots<s_{n}<\right.$ $\left.s_{n+1}=t\right\}$ be a partition of $(s, t)$ of special type $\epsilon$, splitting the interval $(s, t)$ into $(n+1)$ subintervals. Set $m=[(n+1) / 2]$ and $u:=s_{m}$, so the two partitions $\pi_{t u}$ 
and $\pi_{u s}$ are both of special type $\epsilon$, with respective cardinals no greater than $n$. Then we have

$$
\begin{aligned}
& D_{x}\left(\mu_{\pi_{t s}}\right)-D_{x} \mu_{t s}=D_{x}\left(\mu_{\pi_{t u}} \circ \mu_{\pi_{u s}}\right)-D_{x} \mu_{t s} \\
& =\left(D_{\mu_{\pi_{u s}}(x)} \mu_{\pi_{t u}}-D_{\mu_{\pi_{u s}}(x)} \mu_{t u}\right)\left(D_{x} \mu_{\pi_{u s}}\right)+\left(D_{\mu_{\pi_{u s}}(x)} \mu_{t u}-D_{\mu_{u s}(x)} \mu_{t u}\right)\left(D_{x} \mu_{\pi_{u s}}\right) \\
& \quad+\left(D_{\mu_{u s}(x)} \mu_{t u}\right)\left(D_{x} \mu_{\pi_{u s}}-D_{x} \mu_{u s}\right)+\left(\left(D_{\mu_{u s}(x)} \mu_{t u}\right)\left(D_{x} \mu_{u s}\right)-D_{x} \mu_{t s}\right) \\
& =:(1)+(2)+(3)+(4),
\end{aligned}
$$

for any $x \in E$. We treat each term separately using repeatedly the induction hypothesis and Lemma 2.3 when needed. We first have

$$
|(1)| \leqslant L|t-u|^{a}\left(1+o_{\delta}(1)+\delta^{a} K\right) \leqslant\left(1+o_{\delta}(1)\right) L|t-u|^{a} .
$$

Also, using the fact that $|t-u| \leqslant \frac{1-\epsilon}{\epsilon}|u-s|$, we see by (2.1) that we have

$$
\begin{aligned}
\left|D_{\mu_{\pi_{u s}}(x)} \mu_{t u}-D_{\mu_{u s}(x)} \mu_{t u}\right| & \leqslant c|t-u|^{\alpha} L^{\rho}|u-s|^{a \rho}+o_{|t-u|}(1) L|u-s|^{a} \\
& \leqslant o_{\delta}(1)|u-s|^{a},
\end{aligned}
$$

provided $a<\alpha+\rho a$, which we can suppose without loss of generality since $1-\rho<$ $\alpha<1$. As the term $D_{x} \mu_{\pi_{u s}}$ has size no greater than $\left(1+o_{\delta}(1)\right)$, as in the above estimate for (1), we have

$$
|(2)| \leqslant\left(1+o_{\delta}(1)\right) o_{\delta}(1)|u-s|^{a}=o_{\delta}(1)|u-s|^{a} .
$$

Last, we have the upper bound

$$
|(3)| \leqslant\left(1+o_{\delta}(1)\right) L|u-s|^{a},
$$

while $|(4)| \leqslant c_{1}|t-s|^{a}$, by (2.2). All together, and writing $t-u=\beta(t-s)$, with $\epsilon \leqslant \beta \leqslant 1-\epsilon$, this gives

$$
\left|D_{x}\left(\mu_{\pi_{t s}}\right)-D_{x} \mu_{t s}\right| \leqslant\left(\left(1+o_{\delta}(1)\right)\left(\beta^{a}+(1-\beta)^{a}\right) L+c_{1}+o_{\delta}(1)\right)|t-s|^{a} .
$$

The induction is closed by choosing $\delta$ small enough, as a consequence of our choice of constant $L$.

Note in particular that this lemma implies that the maps $\mu_{\pi_{t s}}$ are uniformly Lipschitz continuous; there is no loss of generality in choosing $\delta$ small enough for all the $\mu_{\pi_{t s}}$ to have Lipschitz size smaller than 2 say. Equipped with this lemma, we are in a position to provide a simple proof of Theorem 2.1.

Proof of Theorem 2.1. The existence and uniqueness proofs of the statement of Theorem 2.1 both rely on the elementary identity

$$
f_{N} \circ \cdots \circ f_{1}-g_{N} \circ \cdots \circ g_{1}
$$

$$
=\sum_{i=1}^{N}\left(g_{N} \circ \cdots \circ g_{N-i+1} \circ f_{N-i}-g_{N} \circ \cdots \circ g_{N-i+1} \circ g_{N-i}\right) \circ f_{N-i-1} \circ \cdots \circ f_{1} \text {, }
$$


where the $g_{i}$ and $f_{i}$ are maps from $E$ to itself, and where we use the obvious convention concerning the summand for the first and last term of the sum. In particular, if all the maps $g_{N} \circ \cdots \circ g_{k}$ are Lipschitz continuous, with a common upper bound $c^{\prime}$ for their Lipschitz constants, then

$$
\left\|f_{N} \circ \cdots \circ f_{1}-g_{N} \circ \cdots \circ g_{1}\right\|_{\infty} \leqslant c^{\prime} \sum_{i=1}^{N}\left\|f_{i}-g_{i}\right\|_{\infty} .
$$

a) Existence. Set $D_{\delta}:=\{0 \leqslant s \leqslant t \leqslant T ; t-s \leqslant \delta\}$ and write $\mathbb{D}_{\delta}$ for the intersection of $\mathrm{D}_{\delta}$ with the set of dyadic real numbers. Given $s=a 2^{-n_{0}}$ and $t=b 2^{-n_{0}}$ in $\mathbb{D}_{\delta}$, define for $n \geqslant n_{0}$

$$
\mu_{t s}^{(n)}:=\mu_{t s}^{2^{n}}=\mu_{s_{N(n)} s_{N(n)-1}} \circ \cdots \circ \mu_{s_{1} s_{0}},
$$

where $s_{i}=s+i 2^{-n}$ and $s_{N(n)}=t$. Given $n \geqslant n_{0}$, write

$$
\mu_{t s}^{(n+1)}=\bigodot_{i=0}^{N(n)-1}\left(\mu_{s_{i+1} s_{i}+2^{-n-1}} \circ \mu_{s_{i}+2^{-n-1}} s_{i}\right)
$$

and use (2.9) with $f_{i}=\mu_{s_{i+1} s_{i}+2^{-n-1}} \circ \mu_{s_{i}+2^{-n-1} s_{i}}$ and $g_{i}=\mu_{s_{i+1} s_{i}}$ and the fact that all the maps $\mu_{s_{N(n)} s_{N(n)-1}} \circ \cdots \circ \mu_{s_{N(n)-i+1} s_{N(n)-i}}=\mu_{s_{N(n)} s_{N(n)-i}}$ are Lipschitz continuous, with Lipschitz norm no greater than 2, to get by (2.10) and (2.2)

$$
\begin{aligned}
\left\|\mu_{t s}^{(n+1)}-\mu_{t s}^{(n)}\right\|_{\infty} & \leqslant 2 \sum_{i=0}^{N(n)-1}\left\|\mu_{s_{i+1} s_{i}+2^{-n-1}} \circ \mu_{s_{i}+2^{-n-1} s_{i}}-\mu_{s_{i+1} s_{i}}\right\|_{\infty} \\
& \leqslant 2 c_{1} T 2^{-(a-1) n} ;
\end{aligned}
$$

so $\mu^{(n)}$ converges uniformly on $\mathbb{D}_{\delta}$ to some continuous function $\varphi$. We see that $\varphi$ satisfies inequality $(2.3)$ on $\mathbb{D}_{\delta}$ as a consequence of (2.6). As $\varphi$ is a uniformly continuous function of $(s, t) \in \mathbb{D}_{\delta}$, by $(2.3)$, it has a unique continuous extension to $\mathrm{D}_{\delta}$, still denoted by $\varphi$. To see that it defines a flow on $\mathrm{D}_{\delta}$, notice that for dyadic times $s \leqslant u \leqslant t$, we have $\mu_{t s}^{(n)}=\mu_{t u}^{(n)} \circ \mu_{u s}^{(n)}$, for $n$ big enough; so $\varphi_{t s}=\varphi_{t u} \circ \varphi_{u s}$ for such triples of times in $\mathbb{D}_{\delta}$, hence for all times since $\varphi$ is continuous. The map $\varphi$ is easily extended as a flow to the whole of $\left\{(s, t) \in[0, T]^{2}, 0 \leqslant s \leqslant t \leqslant T\right\}$.

b) Uniqueness. Let $\psi$ be any flow satisfying condition (2.3). With formulas (2.9) and (2.10) in mind, rewrite (2.3) under the form $\psi_{t s}=\mu_{t s}+O_{c}\left(|t-s|^{a}\right)$, with obvious notations. Then

$$
\begin{aligned}
\psi_{t s} & =\psi_{s_{2^{n} s_{2}{ }^{-1}}} \circ \cdots \circ \psi_{s_{1} s_{0}} \\
& =\left(\mu_{s_{2^{n} s_{2^{n}-1}}}+O_{c}\left(2^{-a n}\right)\right) \circ \cdots \circ\left(\mu_{s_{1} s_{0}}+O_{c}\left(2^{-a n}\right)\right) \\
& =\mu_{s_{2^{n} s_{2^{n}-1}}} \circ \cdots \circ \mu_{s_{1} s_{0}}+\Delta_{n}=\mu_{t s}^{(n)}+\Delta_{n},
\end{aligned}
$$

where $\Delta_{n}$ is of the form of the right hand side of (2.9), and so it is bounded above by a constant multiple of $2^{-(a-1) n}$, since all the maps $\mu_{s_{2^{n}} s_{2^{n}-1}} \circ \cdots \circ \mu_{s_{2^{n}-\ell+1} s_{2^{n}}-\ell}$ are Lipschitz continuous with a common upper bound for their Lipschitz constants. Sending $n$ to infinity shows that $\psi_{t s}=\varphi_{t s}$. 
c) Speed of convergence. Given any partition $\pi_{t s}=\left\{s_{0}=s<\cdots<\right.$ $\left.s_{n}=t\right\}$ of $(s, t)$, using as above the uniform Lipschitz character of the maps $\mu_{s_{n} s_{n-1}} \circ \cdots \circ \mu_{s_{i+s} s_{i}}$, and writing $\varphi_{t s}=\bigcirc_{i=0}^{n-1} \varphi_{s_{i+1} s_{i}}$, we see as a consequence of (2.10) that, for $\left|\pi_{t s}\right| \leqslant \delta$,

$$
\begin{aligned}
\left\|\varphi_{t s}-\mu_{\pi_{t s}}\right\|_{\infty} & \leqslant 2 \sum_{i=0}^{n-1}\left\|\varphi_{s_{i+1} s_{i}}-\mu_{s_{i+1}} s_{i}\right\|_{\infty} \\
& \leqslant 2 c_{1} \sum_{i=0}^{n-1}\left|s_{i+1}-s_{i}\right|^{a} \leqslant 2 c_{1} T\left|\pi_{t s}\right|^{a-1}
\end{aligned}
$$

As a straightforward application of Theorem 2.1, it is elementary to see that one defines a $\mathcal{C}^{1}$ approximate flow by setting

$$
\mu_{t s}(x)=x+\left(h_{t}-h_{s}\right)^{i} V_{i}(x)
$$

where $h:[0, T] \rightarrow \mathbb{R}^{\ell}$ is a $\mathcal{C}^{1}$ control and the vector fields $V_{1}, \ldots, V_{\ell}$ on $E$ are $\mathcal{C}_{b}^{2}$. Identity $(2.2)$ holds in that case with $a=2$. The associated flow coincides with the flow associated with the controlled ordinary differential equation

$$
\dot{x}_{t}=V_{i}\left(x_{t}\right) \dot{h}_{t}^{i}
$$

as the latter obviously satisfies estimate (2.3).

\section{Flows driven by Hölder weak geometric $p$-rough paths}

Hölder $p$-rough paths, which control the rough differential equation (1.1) and play the role of $h$ in (2.13), are defined in section 3.1. As $\mathbb{R}^{\ell}$-valued paths, they are not regular enough for formula (2.12) to define an approximate flow. The missing bit of information needed to stabilize the situation is a substitute of the non-existing iterated integrals $\int_{s}^{t} X_{r}^{j} d X_{r}^{k}$, and higher order iterated integrals, which provide a partial description of what happened to $X$ during any time interval $(s, t)$. The higher order parts of a $p$-rough path provide precisely that information. It is an important fact that $p$-rough paths take values in a very special kind of algebraic structure, of which we recall the basic features in section 3.1. We shall then see in section 3.2 how to associate to a rough path and some smooth enough vector fields a $\mathcal{C}^{1}$-approximate flow.

\subsection{An algebraic prelude: tensor algebra over $\mathbb{R}^{\ell}$ and free nilpotent Lie group}

For $N \in \mathbb{N} \cup\{\infty\}$, write $T_{\ell}^{(N)}$ for the direct sum $\bigoplus_{r=0}^{N}\left(\mathbb{R}^{\ell}\right)^{\otimes r}$, with the convention that $\left(\mathbb{R}^{\ell}\right)^{\otimes 0}$ stands for $\mathbb{R}$. Denote by $\mathbf{a}=\oplus_{r=0}^{N} a_{r}$ and $\mathbf{b}=\oplus_{r=0}^{N} b_{r}$ two generic elements of $T_{\ell}^{(N)}$. 
The vector space $T_{\ell}^{(N)}$ is an algebra for the operations

$$
\begin{aligned}
& \mathbf{a}+\mathbf{b}=\underset{r=0}{\oplus}\left(a_{r}+b_{r}\right), \\
& \mathbf{a b}=\underset{r=0}{\oplus} c_{r}, \quad \text { with } \quad c_{r}=\sum_{k=0}^{r} a_{k} \otimes b_{r-k}
\end{aligned}
$$

It is called the (truncated) tensor algebra of $\mathbb{R}^{\ell}$ (if $N$ is finite).

The exponential map exp : $T_{\ell}^{(\infty)} \rightarrow T_{\ell}^{(\infty)}$ and the logarithm map log: $T_{\ell}^{(\infty)} \rightarrow$ $T_{\ell}^{(\infty)}$ are defined by the usual series

$$
\exp (\mathbf{a})=\sum_{n \geqslant 0} \frac{\mathbf{a}^{n}}{n !}, \quad \log (\mathbf{b})=\sum_{n \geqslant 1} \frac{(-1)^{n}}{n}(1-\mathbf{b})^{n},
$$

with the convention $a^{0}=1 \in \mathbb{R} \subset T_{\ell}^{(\infty)}$. Denote by $\pi_{N}: T_{\ell}^{(\infty)} \rightarrow T_{\ell}^{(N)}$ the natural projection. We also denote by $\exp$ and $\log$ the restrictions to $T_{\ell}^{(N)}$ of the maps $\pi_{N} \circ \exp$ and $\pi_{N} \circ \log$ respectively. Denote by $T_{\ell}^{(N), 1}$, resp. $T_{\ell}^{(N), 0}$, the elements $a_{0} \oplus \cdots \oplus c_{N}$ of $T_{\ell}^{(N)}$ such that $a_{0}=0$, resp. $a_{0}=1$. All the elements of $T_{\ell}^{(N), 1}$ are invertible, and $\exp : T_{\ell}^{(N), 0} \rightarrow T_{\ell}^{(N), 1}$ and $\log : T_{\ell}^{(N), 1} \rightarrow T_{\ell}^{(N), 0}$ are reciprocal bijections.

The formula $[\mathbf{a}, \mathbf{b}]=\mathbf{a b}-\mathbf{b a}$, defines a Lie bracket on $T_{\ell}^{(N)}$. Define inductively $F=F^{1}=\mathbb{R}^{\ell}$, considered as a subset of $T_{\ell}^{(\infty)}$, and $F^{n+1}=\left[F, F^{n}\right] \subset T_{\ell}^{(\infty)}$.

Definition 3.1. (a) The Lie algebra $\mathfrak{g}_{\ell}^{N}$ generated by the $F^{1}, \ldots, F^{N}$ is called the $N$-step free nilpotent Lie algebra.

(b) As a consequence of the Baker-Campbell-Hausdorff-Dynkin formula, the subset $\exp \left(\mathfrak{g}_{\ell}^{N}\right)$ of $T_{\ell}^{(N)}$ is a group for the multiplication operation. It is called the $N$-step nilpotent Lie group on $\mathbb{R}^{\ell}$ and denoted by $G_{\ell}^{(N)}$.

Note that the restriction to $G_{\ell}^{(q)}$ of the projection map $\pi_{p q}: T_{\ell}^{(q)} \rightarrow T_{\ell}^{(p)}$, sending $a_{0} \oplus \cdots \oplus a_{q}$ to $a_{0} \oplus \cdots \oplus a_{p}$, provides a natural projection $\pi_{p q}$ from $G_{\ell}^{(q)}$ to $G_{\ell}^{(p)}$ for any $p<q$.

The relevance of the algebraic framework provided by the $N$-step nilpotent Lie group for the study of smooth paths was first noted by Chen in his seminal work [8]. Indeed, for any $\mathbb{R}^{\ell}$-valued smooth path $\left(x_{s}\right)_{s \geqslant 0}$, the family of iterated integrals

$$
\mathfrak{X}_{t s}^{N}:=\left(1, x_{t}-x_{s}, \int_{s}^{t} \int_{s}^{s_{1}} d x_{s_{2}} \otimes d x_{s_{1}}, \ldots, \int_{s \leqslant s_{1} \leqslant \cdots \leqslant s_{N} \leqslant t} d x_{s_{1}} \otimes \cdots \otimes d x_{s_{N}}\right)
$$

defines for all $0 \leqslant s \leqslant t$ an element of $G_{\ell}^{(N)}$. It suffices to notice that, as a function of $t$, the function $\mathfrak{X}_{t s}^{N}$ satisfies the differential equation

$$
d \mathfrak{X}_{t s}^{N}=\mathfrak{X}_{t s}^{N} \otimes d x_{t},
$$


in $T_{\ell}^{(N)}$. Rough paths and weak geometric rough paths are somehow an abstract version of these objects.

Definition 3.2. Let $2 \leqslant p$. A Hölder $p$-rough path on $[0, T]$ is a $T_{\ell}^{([p]), 1}$-valued path $\mathbf{X}: t \in[0, T] \mapsto 1 \oplus X_{t}^{1} \oplus X_{t}^{2} \oplus \cdots \oplus X_{t}^{[p]}$ such that

$$
\sup _{0 \leqslant s<t \leqslant T} \frac{\left|X_{t s}^{i}\right|}{|t-s|^{i / p}}<\infty,
$$

for all $i=1 \ldots[p]$, where we set $\mathbf{X}_{t s}=\mathbf{X}_{s}^{-1} \mathbf{X}_{t}$. We define the norm of $\mathbf{X}$ to be

$$
\|\mathbf{X}\|=\max _{i=1 \ldots[p]} \sup _{0 \leqslant s<t \leqslant T} \frac{\left|X_{t s}^{i}\right|}{|t-s|^{i / p}},
$$

and a distance $d(\mathbf{X}, \mathbf{Y})=\|\mathbf{X}-\mathbf{Y}\|$ on the set of Hölder $p$-rough path. A Hölder weak geometric $p$-rough path on $[0, T]$ is a $G_{\ell}^{([p])}$-valued $p$-rough path.

For $2 \leqslant p<3$, the relation $\mathbf{X}_{u s} \mathbf{X}_{t u}=\mathbf{X}_{t s}$, for $0 \leqslant s \leqslant u \leqslant t \leqslant T$, is equivalent to

(i) $X_{t s}^{1}=X_{t u}^{1}+X_{u s}^{1}$,

(ii) $X_{t s}^{2}=X_{t u}^{2}+X_{u s}^{1} \otimes X_{t u}^{1}+X_{u s}^{2}$.

Condition (i) means that $X_{t s}^{1}=X_{t 0}^{1}-X_{s 0}^{1}$ represents the increment of the $\mathbb{R}^{d}$-valued path $\left(X_{r 0}^{1}\right)_{0 \leqslant r \leqslant T}$. Condition (ii) is nothing but the analogue of the elementary property $\int_{s}^{t} \int_{s}^{r}=\int_{s}^{u} \int_{s}^{r}+\int_{u}^{t} \int_{s}^{u}+\int_{u}^{t} \int_{u}^{r}$, satisfied by any reasonable notion of integral on $\mathbb{R}$ such that $\int_{s}^{t}=\int_{s}^{u}+\int_{u}^{t}$. This remark justifies thinking of the $\left(\mathbb{R}^{\ell} \otimes \mathbb{R}^{\ell}\right)$-part of a rough path as a kind of iterated integral of $X^{1}$ against itself. In that setting, a $p$-rough path $\mathbf{X}$ is a weak geometric $p$-rough path iff the symmetric part of $X_{t s}^{2}$ is $\frac{1}{2} X_{t s}^{1} \otimes X_{t s}^{1}$, for all $0 \leqslant s \leqslant t \leqslant T$.

Remark 3.3. Hölder $p$-rough paths appear naturally in a probabilistic context. Let $B$ be a Brownian motion; the random process

$$
\mathbf{B}=\left(B_{t}-B_{s}, \int_{s}^{t} B_{r} \otimes \circ d B_{r}\right)_{0 \leqslant s \leqslant t \leqslant T}
$$

is almost-surely a weak geometric Hölder $p$-rough path, for any $2<p<3$. It is called the Brownian rough path. Note that using an Itô integral, the formula $\mathbf{B}^{\mathrm{I}}=\left(B_{t}-B_{s}, \int_{s}^{t} B_{r} \otimes d B_{r}\right)_{0 \leqslant s \leqslant t \leqslant T}$, defines a Hölder $p$-rough path which is not weak geometric.

Denote by $\left(e_{1}, \ldots, e_{\ell}\right)$ the canonical basis of $\mathbb{R}^{\ell} \subset T_{\ell}^{(\infty)}$ and write for a tuple $I=\left(i_{1}, \ldots, i_{r}\right)$

$$
\mathbf{e}_{[I]}=\left[e_{i_{1}},\left[e_{i_{2}}, \ldots\left[e_{i_{r-1}}, e_{i_{r}}\right]\right] \ldots\right], \quad \text { and } \mathbf{e}_{I}=e_{i_{1}} e_{i_{2}} \cdots e_{i_{r}}
$$

where the above products are in $T_{\ell}^{(\infty)}$. Write $Y^{r, I}$, with $|I|=r$, or simply $Y^{I}$, for the coordinates of an element $Y$ of $T_{\ell}^{[p]}$ in its canonical basis. 
Given a Hölder weak geometric $p$-rough path $\mathbf{X}$, denote by $\boldsymbol{\Lambda}=0 \oplus \Lambda^{1} \oplus \cdots \oplus \Lambda^{[p]}$ its logarithm, in Magnus-Chen-Strichartz form [22],

$$
\exp \left(\Lambda_{t s}^{I} \mathbf{e}_{[I]}\right)=X_{t s}^{I} \mathbf{e}_{I}=\mathbf{X}_{t s}
$$

for all $0 \leqslant s \leqslant t \leqslant T$; it takes values in the finite dimensional Lie algebra $\mathfrak{g}_{\ell}^{[p]}$. Notice that since $\boldsymbol{\Lambda}$ is polynomial in $\mathbf{X}$, by formula (3.2), it is a continuous function of $\mathbf{X}$.

\subsection{Flows driven by Hölder weak geometric $p$-rough paths}

We have seen in section 2 that a $\mathcal{C}^{1}$-approximate flow on a Banach space $E$ defines a unique flow $\left(\varphi_{t s}\right)_{0 \leqslant s \leqslant t \leqslant T}$ on $E$ such that the inequality

$$
\left\|\varphi_{t s}-\mu_{t s}\right\|_{\infty} \leqslant c|t-s|^{a}
$$

holds for some positive constants $c$ and $a>1$, for all $0 \leqslant s \leqslant t \leqslant T$ sufficiently close. The construction of $\varphi$ is actually quite explicit, for if we denote by $\mu_{\pi_{t s}}$ the composition of the maps $\mu_{t_{i+1} t_{i}}$ along the times $t_{i}$ of a partition $\pi_{t s}$ of an interval $[s, t]$, the map $\mu_{t s}$ satisfies the estimate

$$
\left\|\varphi_{t s}-\mu_{\pi_{t s}}\right\|_{\infty} \leqslant 2 c_{1} T\left|\pi_{t s}\right|^{a-1},
$$

where $c_{1}$ is the constant that appears in the definition of a $\mathcal{C}^{1}$-approximate flow

$$
\left\|\mu_{t u} \circ \mu_{u s}-\mu_{t s}\right\|_{\mathcal{C}^{1}} \leqslant c_{1}|t-s|^{a} .
$$

It follows in particular from equation (3.6) that if $\mu$ depends continuously on some metric space-valued parameter $\lambda$, with respect to the $\mathcal{C}^{0}$-topology, and if identity (3.8) holds uniformly for $\lambda$ moving in a bounded set say, then $\varphi$ depends continuously on $\lambda$, as a uniform limit of continuous functions.

The point about the machinery of $\mathcal{C}^{1}$-approximate flows is that they actually pop up naturally in a number of situations, under the form of a local in time description of the dynamics under study; nothing else than a kind of Taylor expansion. This was quite clear in the example of the ordinary controlled differential equation

$$
d x_{t}=V_{i}\left(x_{t}\right) d h_{t}^{i},
$$

with $\mathcal{C}^{1}$ real-valued controls $h^{1}, \ldots, h^{\ell}$ and $\mathcal{C}_{b}^{2}$ vector fields $V_{1}, \ldots, V_{\ell}$ in $\mathbb{R}^{d}$. The 1-step Euler scheme

$$
\mu_{t s}(x)=x+\left(h_{t}^{i}-h_{s}^{i}\right) V_{i}(x)
$$

defines in that case a $\mathcal{C}^{1}$-approximate flow which has the awaited Taylor-type expansion, in the sense that one has

$$
f\left(\mu_{t s}(x)\right)=f(x)+\left(h_{t}^{i}-h_{s}^{i}\right)\left(V_{i} f\right)(x)+O\left(|t-s|^{>1}\right)
$$

for any function $f$ of class $\mathcal{C}_{b}^{2}$; but $\mu$ fails to be a flow. Its associated flow (by our machinery) is not only a flow, it also satisfies equation (3.10) as a consequence of identity (3.6). 
We shall proceed in a very similar way to give some meaning and solve the rough differential equation on flows

$$
d \varphi=V d t+\mathrm{F} \mathbf{X}(d t)
$$

where $V$ is a Lipschitz continuous vector field on $E$ and $\mathrm{F}=\left(V_{1}, \ldots, V_{\ell}\right)$ is a collection of sufficiently regular vector fields on $\mathrm{E}$, and $\mathbf{X}$ is a Hölder $p$-rough path over $\mathbb{R}^{\ell}$. A solution flow to equation (3.11) will be defined as a flow on $E$ with a uniform Taylor-Euler expansion of the form

$$
f\left(\varphi_{t s}(x)\right)=f(x)+\sum_{|I| \leqslant[p]} X_{t s}^{I}\left(V_{I} f\right)(x)+O\left(|t-s|^{>1}\right),
$$

where $I=\left(i_{1}, \ldots, i_{k}\right) \in \llbracket 1, \ell \rrbracket^{k}$ is a multi-index with size $k \leqslant[p]$, and $X_{t s}^{I}$ stands for the coordinates of $\mathbf{X}_{t s}$ in the canonical basis of $T_{\ell}^{([p]), 1}$. The vector field $V_{i}$ is seen here as a $1^{\text {st }}$-order differential operator, and $V_{I}=V_{i_{1}} \cdots V_{i_{k}}$ as the $k^{\text {th }}$-order differential operator obtained by applying successively the operators $V_{i_{n}}$.

For $V=0$ and $\mathbf{X}$ the weak geometric Hölder $p$-rough path canonically associated with an $\mathbb{R}^{\ell}$-valued $\mathcal{C}^{1}$ control $h$, with $2 \leqslant p<3$, equation (3.12) becomes

$$
\begin{aligned}
f\left(\varphi_{t s}(x)\right)= & f(x)+\left(h_{t}^{i}-h_{s}^{i}\right)\left(V_{i} f\right)(x) \\
& +\left(\int_{s}^{t} \int_{s}^{r} d h_{u}^{j} d h_{r}^{k}\right)\left(V_{j} V_{k} f\right)(x)+O\left(|t-s|^{>2}\right),
\end{aligned}
$$

which is nothing else than Taylor formula at order 2 for the solution to the ordinary differential equation (3.9) started at $x$ at time $s$. Condition (3.12) is a natural analogue of (3.13) and its higher order analogues.

There is actually a simple way of constructing a map $\mu_{t s}$ which satisfies the Euler expansion (3.12). It can be defined as the time 1 map associated with an ordinary differential equation constructed form the $V_{i}$ and their brackets, and where $\mathbf{X}_{t s}$ appears as a parameter under the form of its logarithm. That these maps $\mu_{t s}$ form a $\mathcal{C}^{1}$-approximate flow will eventually appear as a consequence of the fact that the time 1 map of a differential equation formally behaves as an exponential map, in some algebraic sense. In the sequel, $p$ and $\gamma$ will always denote real numbers such that

$$
1 \leqslant p<\gamma \leqslant[p]+1
$$

Given a bounded Lipschitz continuous vector field $V$, and some $[\gamma]$-Lipschitz vector fields $V_{1}, \ldots, V_{\ell}$ on $E$, let $\mu_{t s}$ be the well-defined time 1 map associated with the ordinary differential equation

$$
\dot{y}_{u}=(t-s) V\left(y_{u}\right)+\sum_{r=1}^{[p]} \sum_{I \in \llbracket 1, \ell \rrbracket^{r}} \Lambda_{t s}^{r, I} V_{[I]}\left(y_{u}\right), \quad 0 \leqslant u \leqslant 1 .
$$

This equation is to be understood as the classical ordinary differential equation version of equation (1.1); the definition of a solution flow to equation (1.1) given 
below will make that point clear. The property of $\mu_{t s}$ proved in Proposition 3.5 below is the main reason for its introduction; it roughly says that $\mu_{t s}$ has the awaited Euler expansion, in accordance with what happens for ordinary differential equations, as emphasized in equation (3.13). For $2 \leqslant p<3$, and $\mathbf{X}=(X, \mathbb{X})$, equation (3.14) reads

$$
\dot{y}_{u}=(t-s) V\left(y_{u}\right)+X_{t s}^{i} V_{i}\left(y_{u}\right)+\frac{1}{2}\left\{\mathbb{X}_{t s}+\frac{1}{2} X_{t s} \otimes X_{t s}\right\}^{j k}\left[V_{j}, V_{k}\right]\left(y_{u}\right) .
$$

As the matrix $X_{t s} \otimes X_{t s}$ is symmetric, we have $X_{t s}^{j} X_{t s}^{k}\left[V_{j}, V_{k}\right]=0$, so (3.14) simplifies into

$$
\dot{y}_{u}=(t-s) V\left(y_{u}\right)+X_{t s}^{i} V_{i}\left(y_{u}\right)+\frac{1}{2} \mathbb{X}_{t s}^{j k}\left[V_{j}, V_{k}\right]\left(y_{u}\right) .
$$

If the vector field $V$ is $\mathcal{C}^{1}$ and the $V_{1}, \ldots, V_{\ell}$ are $\gamma$-Lipschitz, the classical results on the dependence of solutions to ordinary differential equation with respect to some parameters ensure that for any reals numbers $a, b^{I}$, the time 1 map $\exp \left(a V+b^{I} V_{[I]}\right)$ associated with the differential equation

$$
\frac{d}{d u} y_{u}=a V\left(y_{u}\right)+b^{I} V_{[I]}\left(y_{u}\right), \quad 0 \leqslant u \leqslant 1,
$$

is a continuously differentiable function of $(a, b)$. The following basic fact comes as a consequence of the analytic properties of any Hölder $p$-rough path and the definition of the topology on the set of Hölder $p$-rough paths. We write $\mu_{t s}^{\mathbf{X}}$ instead of $\mu_{t s}$ in the proposition below to emphasize its dependence on $\mathbf{X}$.

Proposition 3.4. Let $V$ be Lipschitz continuous and the $V_{i}$ be $[\gamma]$-Lipschitz.

(1) Then all the maps $\mu_{t s}^{\mathbf{X}}$ are uniformly Lipschitz continuous, with Lipschitz constant no greater than $1+c|t-s|^{1 / p}$, for a constant $c=c^{\prime}\left(1+\|\mathbf{X}\|^{\gamma}\right)$, with $c^{\prime}$ depending on the data of the problem.

(2) The Lipschitz homeomorphisms $\mu_{t s}^{\mathbf{X}}$ depend continuously on $((s, t), \mathbf{X})$, in the sense that

$$
\max \left\{\left\|\mu_{t s}^{\mathbf{X}}-\mu_{v u}^{\mathbf{Y}}\right\|_{\infty},\left\|\left(\mu_{t s}^{\mathbf{X}}\right)^{-1}-\left(\mu_{v u}^{\mathbf{Y}}\right)^{-1}\right\|_{\infty}\right\} \leqslant f((s, t),(u, v) ; \mathbf{X}, \mathbf{Y})
$$

for a continuous function $f$ with $f((s, t),(s, t) ; \mathbf{X}, \mathbf{X})=0$, for all $0 \leqslant s \leqslant$ $t \leqslant T$, and any Hölder p-rough path $\mathbf{X}$.

The following proposition is our basic step for studying flows driven by rough paths.

Proposition 3.5. Let $0<\rho<1$ and $2 \leqslant p<\gamma<[p]+1$ be given. Let $V$ be a Lipschitz-continuous vector field on $E$, and $V_{1}, \ldots, V_{\ell}$ be $[\gamma]$-Lipschitz vector fields on E. Let $\mathbf{X}=1 \oplus X^{1} \oplus \cdots \oplus X^{[p]}$ be a Hölder weak geometric p-rough path. 
Then there exists a positive constant $c$, depending only on the data of the problem, such that

$$
\begin{aligned}
\| f \circ \mu_{t s}-\left\{f+(t-s) V f+\sum_{r=1}^{[p]}\right. & \left.\sum_{I \in \llbracket 1, \ell \rrbracket^{r}} X_{t s}^{r, I} V_{I} f\right\} \|_{\infty} \\
& \leqslant c\left(1+\|\mathbf{X}\|^{\gamma}\right)\|f\|_{\gamma}|t-s|^{\gamma / p}
\end{aligned}
$$

holds for any $f \in \mathcal{C}^{\gamma}$.

The proof of this proposition and the following one are based on the following elementary identity, obtained by applying repeatedly the identity

$$
f\left(y_{r}\right)=f(x)+(t-s) \int_{0}^{1}(V f)\left(y_{u}\right) d u+\sum_{I} \Lambda_{t s}^{I} \int_{0}^{1}\left(V_{[I]} f\right)\left(y_{u}\right) d u
$$

and by separating the terms according to their size in $|t-s|$. Set

$$
\Delta_{n}:=\left\{\left(s_{1}, \ldots, s_{n}\right) \in[0, T]^{n} ; s_{1} \leqslant \cdots \leqslant s_{n}\right\},
$$

for $2 \leqslant n \leqslant[p]$ and write $d s$ for Lebesgue volume element on $\Delta_{n}$. For a $\gamma$-Lipschitz function $f$, we have

$$
\begin{aligned}
& f\left(\mu_{t s}(x)\right)=f(x)+(t-s)(V f)(x) \\
& +\sum_{k=1}^{n} \frac{1}{k !} \sum_{\left|I_{1}\right|+\cdots+\left|I_{k}\right| \leqslant[p]}\left(\prod_{m=1}^{k} \Lambda_{t s}^{I_{m}}\right)\left(V_{\left[I_{k}\right]} \cdots V_{\left[I_{1}\right]} f\right)(x) \\
& +\sum_{\left|I_{1}\right|+\cdots+\left|I_{n}\right| \leqslant[p]}\left(\prod_{m=1}^{n} \Lambda_{t s}^{I_{m}}\right) \int_{\Delta_{n}}\left\{\left(V_{\left[I_{n}\right]} \cdots V_{\left[I_{1}\right]} f\right)\left(y_{s_{n}}\right)-\left(V_{\left[I_{n}\right]} \cdots V_{\left[I_{1}\right]} f\right)(x)\right\} \mathbf{1}_{\Delta_{n}} d s \\
& +(t-s) \int_{0}^{1}\left\{(V f)\left(y_{r}\right)-(V f)(x)\right\} d r \\
& +\sum_{k=1}^{n-1} \frac{1}{k !} \sum_{\left|I_{1}\right|+\cdots+\left|I_{k}\right| \geqslant[p]+1}\left(\prod_{m=1}^{k} \Lambda_{t s}^{I_{m}}\right)\left(V_{\left[I_{k}\right]} \cdots V_{\left[I_{1}\right]} f\right)(x) \\
& +(t-s) \sum_{k=1}^{n-1} \sum_{I_{1}, \ldots, I_{k}}\left(\prod_{m=1}^{k} \Lambda_{t s}^{I_{m}}\right) \int_{\Delta_{k}}\left(V V_{\left[I_{k}\right]} \cdots V_{\left[I_{1}\right]} f\right)\left(y_{s_{k}}\right) \mathbf{1}_{\Delta_{k}} d s_{k} \ldots d s_{1} \\
& +\sum_{\mathfrak{I}_{n, p}}\left(\prod_{m=1}^{k} \Lambda_{t s}^{I_{m}}\right) \int_{\Delta_{n}}\left\{\left(V_{\left[I_{n}\right]} \cdots V_{\left[I_{1}\right]} f\right)\left(y_{s_{n}}\right)-\left(V_{\left[I_{n}\right]} \cdots V_{\left[I_{1}\right]} f\right)(x)\right\} \mathbf{1}_{\Delta_{n}} d s,
\end{aligned}
$$

where the last sum is over the set $\mathfrak{I}_{n, p}$ of tuples $I_{1}, \ldots, I_{n}$ such that

$$
\left|I_{1}\right|+\cdots+\left|I_{n}\right| \geqslant[p]+1
$$


We denote by $\epsilon_{t s}^{f ; n}(x)$ the sum of the last four lines, made up of terms of size at least $|t-s|^{\gamma / p}$. Note that this formula makes sense for all $2 \leqslant n \leqslant[p]$, and for $(\gamma-1)$-Lipschitz vector fields $V_{i}$. In the case where $n=[p]$, the terms in the second line involve only indices $I_{j}$ with $\left|I_{j}\right|=1$, so the elementary estimate

$$
\left|y_{r}-x\right| \leqslant c\left(1+\|\mathbf{X}\|^{\gamma}\right)|t-s|^{1 / p}, \quad 0 \leqslant r \leqslant 1
$$

can be used to control the increment in the integral, showing that this second line is of order $|t-s|^{\gamma / p}$, as the maps $V_{\left[I_{[p]}\right]} \ldots V_{\left[I_{1}\right]} f$ are $(\gamma-[p])$-Lipschitz; we include it in the remainder $\epsilon_{t s}^{f ;[p]}(x)$.

Proof of theorem 3.5. Applying the above formula for $n=[p]$, together with the fact that $\exp (\boldsymbol{\Lambda})=\mathbf{X}$, we get the identity

$$
f\left(\mu_{t s}(x)\right)=f(x)+(t-s)(V f)(x)+\sum_{I} X_{t s}^{I}\left(V_{I} f\right)(x)+\epsilon_{t s}^{f ;[p]}(x) .
$$

It is clear on the formula for $\epsilon_{t s}^{f ;[p]}(x)$ that its absolute value is bounded above by a constant multiple of $\left(1+\|\mathbf{X}\|^{\gamma}\right)|t-s|^{\gamma / p}$, for a constant depending only on the data of the problem and $f$ as in (3.15).

A further look at formula (3.16) also makes it clear that if $V$ is $(1+\rho)$-Lipschitz and the $V_{i}$ are $\gamma$-Lipschitz, with $f$ sufficiently regular, then the remainders $\epsilon_{t s}^{f ; n}, 2 \leqslant$ $n \leqslant[p]$, have the norm of their first derivative bounded above by a constant multiple of $\left(1+\|\mathbf{X}\|^{\gamma}\right)|t-s|^{a}$, for a constant depending only on the data of the problem, and $a=\min \{1+\rho / p, \gamma / p\}$. This is the key remark for proving the next proposition.

Proposition 3.6. (1) Suppose $V$ is Lipschitz-continuous and the $V_{i}$ are $[\gamma]$ Lipschitz. Then $\left(\mu_{t s}\right)_{0 \leqslant s \leqslant t \leqslant T}$ is a $\mathcal{C}^{0}$-approximate flow.

(2) Suppose $V$ is $(1+\rho)$-Lipschitz, for some $\rho>\frac{p-[p]}{p}$, and the $V_{i}$ are $\gamma$-Lipschitz, then $\left(\mu_{t s}\right)_{0 \leqslant s \leqslant t \leqslant T}$ is a $\mathcal{C}^{1}$-approximate flow.

Proof. We first use formula (3.16) to write

$$
\mu_{t u}\left(\mu_{u s}(x)\right)=\mu_{u s}(x)+(t-u) V\left(\mu_{u s}(x)\right)+\sum_{I} X_{t u}^{I} V_{I}\left(\mu_{u s}(x)\right)+\epsilon_{t u}^{\mathrm{Id} ;[p]}\left(\mu_{u s}(x)\right) .
$$

We deal with the term $(t-u) V\left(\mu_{u s}(x)\right)$ using (3.17) and the Lipschitz character of $V$. The remainder $\epsilon_{t u}^{\mathrm{Id} ;[p]}\left(\mu_{u s}(x)\right)$ has, under the assumptions of statement (1), an infinite norm bounded above by $c\left(1+\|\mathbf{X}\|^{\gamma}\right)|t-u|^{a}$, and its derivative has infinite norm bounded above by $c\left(1+\|\mathbf{X}\|{ }^{\gamma}\right)^{2}|t-u|^{a}$, under the assumptions of (2), by the above remark and part (2) of Proposition 3.4 about the uniform Lipschitz size of the maps $\mu_{b a}$.

To deal with the terms $X_{t u}^{I} V_{I}\left(\mu_{u s}(x)\right)$, with $|I|=k$, we use formula (3.16) with $n=([p]-k)$, to develop $V_{I}\left(\mu_{u s}(x)\right)$. Write $d s$ for $d s_{[p]-k} \ldots d s_{1}$ in the formula 
below. We have

$$
\begin{aligned}
V_{I}\left(\mu_{u s}(x)\right)= & V_{I}(x)+(u-s)\left(V V_{I}\right)(x) \\
& +\sum_{j=1}^{[p]-k} \frac{1}{j !} \sum_{\left|I_{1}\right|+\cdots+\left|I_{j}\right| \leqslant[p]}\left(\prod_{m=1}^{j} \Lambda_{u s}^{I_{m}}\right)\left(V_{\left[I_{j}\right]} \cdots V_{\left[I_{1}\right]} V_{I}\right)(x)+\epsilon_{u s}^{V_{I} ; p-k}(x) \\
& +\sum_{\left|I_{1}\right|+\cdots+\left|I_{[p]-k}\right| \leqslant[p]}\left(\prod_{m=1}^{[p]-k} \Lambda_{u s}^{I_{m}}\right) \int_{0}^{1}\left\{\left(V_{\left[I_{[p]-k}\right]} \cdots V_{\left[I_{1}\right]} V_{I}\right)\left(y_{s_{[p]-k}}\right)\right. \\
& \left.-\left(V_{\left[I_{[p]-k}\right]} \cdots V_{\left[I_{1}\right]} V_{I}\right)(x)\right\} \mathbf{1}_{\Delta_{[p]-k}} d s .
\end{aligned}
$$

We see, using (3.17), that $X_{t u}^{I}$ times the second line above has an infinite norm bounded above by $c\left(1+\|\mathbf{X}\|^{\gamma}\right)|t-s|^{a}$, and that its derivative has an infinite norm bounded above by $c\left(1+\|\mathbf{X}\|^{\gamma}\right)^{2}|t-s|^{a}$, under the regularity assumptions of (2).

Writing

$$
\mu_{u s}(x)=x+(u-s) V(x)+\sum_{I} X_{u s}^{I} V_{I}(x)+\epsilon_{u s}^{\mathrm{Id} ;[p]}(x),
$$

it is then straightforward to use the identities $\exp \left(\boldsymbol{\Lambda}_{u s}\right)=\mathbf{X}_{u s}$ and $\mathbf{X}_{t s}=\mathbf{X}_{u s} \mathbf{X}_{t u}$, to see that

$$
\mu_{t u}\left(\mu_{u s}(x)\right)=\mu_{t s}(x)+\epsilon_{t s}(x),
$$

with a remainder $\left\|\epsilon_{t s}\right\|_{\infty} \leqslant c\left(1+\|\mathbf{X}\|^{\gamma}\right)|t-s|^{a}$, under the regularity assumptions of point (1), with first derivative with infinite norm bounded above by $c(1+\|\mathbf{X}\| \gamma)^{2}|t-s|^{a}$, under the assumptions of statement (2).

It remains to check that the decomposition (2.1) holds in that case, which is done by writing

$$
\begin{aligned}
\mu_{t s}(x)= & +\int_{0}^{1}\left\{(t-s) V\left(y_{r}^{x}\right)+\sum_{|I|=[p]} \Lambda_{t s}^{I} V_{[I]}\left(y_{r}^{x}\right)\right\} d r \\
& +\int_{0}^{1} \sum_{|I| \leqslant[p]-1} \Lambda_{t s}^{I} V_{[I]}\left(y_{r}^{x}\right) d r
\end{aligned}
$$

(where we have emphasized the dependence of $y_{r}$ on its initial condition $x$ by an upper index $x$ ), and defining $A_{x}^{t s}$, as the differential of the function of $x$ defined by the first integral, and $B_{x}^{t s}$, by the differential of the function of $x$ defined by the second integral; with the notation of Theorem 2.1, we have $\alpha=[p] / p$ here.

The following is to be thought of as an analogue in the setting of flows of Davie's definition of a solution to a rough differential equation [9], as recalled in the introduction.

Definition 3.7. Let $2 \leqslant p<\gamma<[p]+1$ be given. Let $V_{1}, \ldots, V_{\ell}$ be $\mathcal{C}_{b}^{[p]}$-Lipschitz vector fields on $E$, and $\mathbf{X}$ be a Hölder weak geometric $p$-rough path. Write $F$ for $\left(V_{1}, \ldots, V_{\ell}\right)$. Let $V$ be a bounded Lipschitz continuous vector field on $E$. With 
the above notations, a flow $\left(\varphi_{t s} ; 0 \leqslant s \leqslant t \leqslant T\right)$ is said to solve the rough differential equation

$$
d \varphi=V d t+F \mathbf{X}(d t)
$$

if there exists a constant $a>1$ independent of $\mathbf{X}$ and two possibly $\mathbf{X}$-dependent positive constants $\delta$ and $c$ such that

$$
\left\|\varphi_{t s}-\mu_{t s}\right\|_{\infty} \leqslant c|t-s|^{a}
$$

holds for all $0 \leqslant s \leqslant t \leqslant T$ with $t-s \leqslant \delta$.

The following well-posedness result follows directly from Theorem 2.1 and Proposition 3.6.

Theorem 3.8. Suppose $V$ is $(1+\rho)$-Lipschitz, for some $\rho>\frac{p-[p]}{p}$, and the $V_{i}$ are $\gamma$-Lipschitz. Then the rough differential equation $d \varphi=V d t+\mathrm{F} \mathbf{X}(d t)$ has a unique solution flow; it takes values in the space of uniformly Lipschitz continuous homeomorphisms of $E$ with uniformly Lipschitz continuous inverses, and depends continuously on $\mathbf{X}$.

Proof. Note that any solution flow depends continuously on $(s, t)$ in the topology of uniform convergence on $E$ by Proposition 3.4 and the defining condition (3.20). Use the notation $c_{1}$ of section 2 for the constant appearing in the sewing lemma for flows, and write $a$ for $\min \{1+\rho / p, \gamma / p\}$; it follows from the above estimates that we can choose

$$
c_{1}=c\left(1+\|\mathbf{X}\|^{\gamma}\right)^{2},
$$

so we have

$$
\left\|\varphi_{t s}-\mu_{\pi_{t s}}\right\|_{\infty} \leqslant c\left(1+\|\mathbf{X}\|^{\gamma}\right)^{2} T\left|\pi_{t s}\right|^{a-1},
$$

for any partition $\pi_{t s}$ of $(s, t) \subset[0, T]$ of mesh $\left|\pi_{t s}\right| \leqslant \delta$, as a consequence of inequality (2.4). As these bounds are uniform in $(s, t)$, and for $\mathbf{X}$ in a bounded set of the space of Hölder $p$-rough paths, and each $\mu_{\pi_{t s}}$ is a continuous function of $\mathbf{X}$, by Proposition 3.4, the flow $\varphi$ depends continuously on $((s, t), \mathbf{X})$.

To prove that $\varphi$ is a homeomorphism, note that, with the notations of section 2 ,

$$
\left(\mu_{t s}^{(n)}\right)^{-1}=\mu_{s_{1} s_{0}}^{-1} \circ \cdots \circ \mu_{s_{2} s_{2^{n}}-1}^{-1}
$$

can actually be written $\left(\mu_{t s}^{(n)}\right)^{-1}=\widetilde{\mu}_{s_{2} s_{2^{n}-1}} \circ \cdots \circ \widetilde{\mu}_{s_{1} s_{0}}$, for the time 1 map $\widetilde{\mu}$ associated with the rough path $\mathbf{X}_{t-\bullet}$. As $\widetilde{\mu}$ enjoys the same properties as $\mu$, the maps $\left(\mu_{t s}^{(n)}\right)^{-1}$ converge uniformly to some continuous map $\varphi_{t s}^{-1}$ which satisfies by construction $\varphi_{t s} \circ \varphi_{t s}^{-1}=\mathrm{Id}$.

As Lemma 2.4 provides a uniform control of the Lipschitz norm of the maps $\mu_{t s}^{(n)}$, the limit maps $\varphi_{t s}$ also have Lipschitz norms controlled by the same quantity; the same holds for their inverses. We propagate this property from the set $\{(s, t) \in$ $\left.[0, T]^{2} ; s \leqslant t, t-s \leqslant \delta\right\}$ to the whole of $\left\{(s, t) \in[0, T]^{2} ; s \leqslant t\right\}$ using the flow property of $\varphi$. 
Remarks 3.9. (1) Friz-Victoir approach to rough paths. The continuity of the solution flow with respect to the driving rough path $\mathbf{X}$ has the following consequence, which justifies the point of view adopted by Friz and Victoir in their works. Suppose the Hölder weak geometric $p$-rough path $\mathbf{X}$ is the limit in the rough path metric of the canonical Hölder weak geometric $p$-rough paths $\mathbf{X}^{n}$ associated with a smooth (or Lipschitz continuous) $E$-valued path $\left(x_{t}^{n}\right)_{0 \leqslant t \leqslant T}$ through the data of its well-defined iterated integrals. The illustration to the " $\mathcal{C}$-approximate flow to flow" Theorem 2.1 given at the end of section 2 shows that the solution flow $\varphi^{n}$ to the rough differential equation (3.19) with driving rough path $\mathbf{X}^{n}$ is the flow associated with the ordinary differential equation

$$
\dot{y}_{u}=V\left(y_{u}\right) d u+V_{i}\left(y_{u}\right) d\left(x_{u}^{n}\right)^{i} .
$$

As $\left\|\varphi^{n}-\varphi\right\|_{\infty}=o_{n}(1)$, from the continuity of the solution flow with respect to the driving rough path, the flow $\varphi$ appears in that case as a uniform limit of the elementary flows $\varphi^{n}$. A Hölder weak geometric $p$-rough path with the above property is called a Hölder geometric p-rough path; not all Hölder weak geometric p-rough path are Hölder geometric $p$-rough path [14].

Friz and Victoir obtain in [15] convergence rates similar to (3.21), with a slightly better exponent, by a clever use of sub-Riemannian geometry in the free nilpotent Lie group $G_{\ell}^{[p]}$, for rough differential equations without a drift. The above estimate for rough differential equations with a drift appears to be new.

(2) Time-inhomogeneous dynamics. The above result have a straightforward generalization for a time dependent bounded drift $V(s ; \cdot)$ which is Lipschitz continuous with respect to the time and $(1+\rho)$-Lipschitz with respect to the space variable, and time dependent bounded vector fields $V_{i}(s ; \cdot)$ which are $\gamma$-Lipschitz with respect to the space variable and Lipschitz continuous with respect to time. We define in that case a $\mathcal{C}^{1}$-approximate flow by defining $\mu_{t s}$ as the time 1 map associated with the ordinary differential equation

$$
\dot{y}_{u}=(t-s) V\left(s ; y_{u}\right)+\sum_{r=1}^{[p]} \sum_{I \in \llbracket 1, \ell \rrbracket^{r}} \Lambda_{t s}^{r, I} V_{[I]}\left(s ; y_{u}\right), \quad 0 \leqslant u \leqslant 1 .
$$

In particular, inequality (3.15) holds in that case, with $V(s ; x)$ and $V_{I}(s ; x)$ in place of $V(x)$ and $V_{I}(x)$. This framework will be useful in section 6 for the study of some simple mean field stochastic rough differential equation.

\subsection{High order Taylor expansion of solution flows to rough differential equations}

It is a nice feature of our approach that Taylor expansion of solution flows to rough differential equations come almost for free, providing an alternative and simple proof of similar results due to Friz and Victoir [15]. As in the classical case, the smoother the vector fields $V_{i}$ are, the better we can describe the solution flow to the driftless equation $d \varphi=\mathrm{FX}(d t)$. This takes the form of a refined version of the inequality $\left\|\varphi_{t s}-\mu_{t s}\right\|_{\infty} \leqslant c|t-s|^{\gamma / p}$. 
Let $2 \leqslant p$ and an integer $N \geqslant[p]+1$ be given. Lyons devised in his original theory of rough paths [19] a fundamental mechanism which enables in particular to extend uniquely any Hölder weak geometric $p$-rough path to a very special $G_{\ell}^{(N)}$ valued map and provides the missing higher order iterated integrals needed to write a Taylor expansion of the solution flow to the equation $d \varphi=\mathrm{F} \mathbf{X}(d t)$. We state it here under the form we need - see [11] for a simple proof of a refined version of Lyons' extension theorem.

Theorem 3.10 (Lyons' extension theorem). Let $2 \leqslant p$ be given and $\mathbf{X}$ be a Hölder weak geometric p-rough path. Let $N \geqslant[p]+1$ be an integer. Then there exists a unique $\left(G_{\ell}^{(N)}\right.$-valued) Hölder weak geometric $N$-rough path path $\mathbf{Y}$ extending $\mathbf{X}$ in the sense that $\pi_{[p] N} \mathbf{Y}_{t s}=\mathbf{X}_{t s}$, for all $0 \leqslant s \leqslant t \leqslant T$. The map $\mathbf{Y}$ is called the lift of $\mathbf{X}$ to $G_{\ell}^{(N)}$; it is a continuous function of $\mathbf{X}$.

Given $0<\alpha<1$, set $\gamma=N+\alpha$, and suppose the vector fields $V_{i}$ are $\gamma$-Lipschitz. Given a Hölder weak geometric $p$-rough path $\mathbf{X}$, let $\mathbf{Y}$ its lift to $G_{\ell}^{(N)}$, and $\boldsymbol{\Lambda}=$ $0 \oplus \Lambda^{1} \oplus \Lambda^{2} \oplus \cdots \oplus \Lambda^{N}$ be the logarithm of its lift, so we have $\exp \boldsymbol{\Lambda}_{t s}=\mathbf{Y}_{t s}$, for all $0 \leqslant s \leqslant t \leqslant T$.

Denote by $\nu_{t s}^{[N]}$ the diffeomorphism of $E$ which associates to any $x \in E$ the value at time 1 of the well-defined and unique solution of the ordinary differential equation

$$
\frac{d}{d r} y_{u}=\sum_{r=1}^{N} \sum_{I \in \llbracket 1, \ell \rrbracket^{r}} \Lambda_{t s}^{r, I} V_{[I]}\left(y_{u}\right), \quad 0 \leqslant u \leqslant 1,
$$

with $y_{0}=x$. The proof of Proposition 3.5 shows that the following awaited estimate holds.

Proposition 3.11. Let $2 \leqslant p$ and $[p]+1 \leqslant N<N+\alpha<N+1$ be given, with $N$ integer. Let $V_{1}, \ldots, V_{\ell}$ be $(N+\alpha)$-Lipschitz vector fields on $E$. Let $\mathbf{X}=$ $1 \oplus X^{1} \oplus \cdots \oplus X^{[p]}$ be a Hölder weak geometric $p$-rough path with lift $\mathbf{Y}$ to $G_{\ell}^{(N)}$. Then there exists a positive constant $c$, depending only on $M, \lambda, T$ and $\|\mathbf{X}\|$ and $f \in \mathcal{C}^{\gamma}$ such that

$$
\left\|f \circ \nu_{t s}^{[N]}-\left\{f+\sum_{r=1}^{N} \sum_{I \in \llbracket 1, \ell \rrbracket^{r}} Y_{t s}^{r, I} V_{I} f\right\}\right\|_{\infty} \leqslant c|t-s|^{(N+\alpha) / p}
$$

holds for all $f \in \mathcal{C}^{\gamma}$. The maps $\nu_{t s}^{[N]}$ depend continuously on $((s, t), \mathbf{X})$ in uniform topology.

As in section 3.2, this fundamental estimate implies together with Theorem 2.1 a well-posedness result.

Theorem 3.12. Let $2 \leqslant p$ and $[p]+1 \leqslant N<N+\alpha<N+1$ be given, with $N$ integer, and $V_{1}, \ldots, V_{\ell}$ be $(N+\alpha)$-Lipschitz vector fields on $E$. There exists a 
unique flow $\left(\varphi_{t s}^{[N]}\right)_{0 \leqslant s \leqslant t \leqslant T}$ on $E$ for which there are two positive constants $\delta$ and $c$ such that

$$
\left\|\varphi_{t s}^{[N]}-\nu_{t s}^{[N]}\right\|_{\infty} \leqslant c|t-s|^{(N+\alpha) / p}
$$

holds for all $0 \leqslant s \leqslant t \leqslant T$, with $t-s \leqslant \delta$.

Let now $\gamma \in(p,[p]+1)$. Since $\left\|\nu_{t s}^{[N]}-\mu_{t s}\right\|_{\infty} \leqslant c|t-s|^{\gamma / p}$, the identity

$$
\left\|\varphi_{t s}^{[N]}-\mu_{t s}\right\|_{\infty} \leqslant c|t-s|^{\gamma / p}
$$

holds for all $0 \leqslant s \leqslant t \leqslant T$, sufficiently close, so $\varphi^{[N]}$ is the solution flow to the rough differential equation $d \varphi=\mathrm{FX}(d t)$.

Corollary 3.13 (High order Euler estimates/Taylor expansion). Let $2 \leqslant p$, an integer $[p]+1 \leqslant N$ and $0<\alpha<1$ be given. Suppose the vector fields $V_{1}, \ldots, V_{\ell}$ are $(N+\alpha)$-Lipschitz. Let $\mathbf{X}$ be a Hölder weak geometric p-rough path. Denote by $\varphi$ the unique solution flow to the driftless rough differential equation $d \varphi=\mathrm{F} \mathbf{X}(d t)$. Let $\mathbf{Y}$ be the lift of $\mathbf{X}$ to $G_{\ell}^{(N)}$. Then there exists two positive constants $\delta$ and $c$ such that

$$
\left\|\varphi_{t s}-\nu_{t s}^{[N]}\right\|_{\infty} \leqslant c|t-s|^{(N+\alpha) / p}
$$

holds for all $0 \leqslant s \leqslant t \leqslant T$ with $t-s \leqslant \delta$. We have in particular,

$$
\left\|\varphi_{t s}-\left\{\mathrm{Id}+\sum_{r=1}^{N} \sum_{I \in \llbracket 1, \ell \rrbracket^{r}} Y_{t s}^{r, I} V_{I}\right\}\right\|_{\infty} \leqslant c|t-s|^{(N+\alpha) / p}
$$

for all $0 \leqslant s \leqslant t \leqslant T$, with $t-s \leqslant \delta$.

Remarks 3.14. (1) In a probabilistic setting where $\mathbf{X}$ is the random realization of the Brownian rough path, the $Y_{t s}^{k, I}$ coincide almost-surely with the iterated Stratonovich integrals $\int_{0}^{t} \circ d B_{s_{1}}^{i_{1}} \otimes \cdots \otimes \circ d B_{s_{k}}^{i_{k}}$, for $I=\left(i_{1}, \ldots, i_{k}\right)$, and (3.24) is a pathwise version of Azencott's celebrated stochastic Taylor formula - see for instance [1], [4] and [7].

(2) Friz and Victoir [15] proved similar estimates by a clever use of geodesic approximation in the free nilpotent Lie group $G_{\ell}^{(N)}$.

(3) The above theorem provides a straightforward justification of Friz and Oberhauser's theorem about drift induced by perturbed driving signals; see Theorem 2 in $[13]$.

\section{Non-explosion under linear growth conditions on the vec- tor fields}

Let $1<\gamma$ be a non-integer real number. Let us say that a vector field $W$ is $\gamma$-Lipschitz with linear growth if it is $\mathcal{C}^{[\gamma]}$, with bounded derivatives

$$
W^{(1)}, \ldots, W^{([\gamma])}
$$

and its $[\gamma]$ 's derivative is a bounded $(\gamma-[\gamma])$-Hölder map. We do not require that $W$ itself be bounded. We describe in that section how the arguments of sections 2 
and 3 need to be amended to prove a well-posedness result for the rough differential equation on flows (1.1), under the relaxed assumption that the driving vector fields $V, V_{1}, \ldots, V_{\ell}$ are sufficiently regular and have linear growth. We need for that purpose a suitable version of the sewing lemma for flows which applies in that setting. The main difficulty here is that the solution to the ordinary differential equation

$$
\dot{y}_{u}=(t-s) V\left(y_{u}\right)+\sum_{r=1}^{[p]} \sum_{I \in \llbracket 1, \ell \rrbracket^{r}} \Lambda_{t s}^{r, I} V_{[I]}\left(y_{u}\right)
$$

may explode in finite time since the vector fields $V_{[I]}$ have polynomial growth now. This tendency is compensated by the small size of the coefficients $\Lambda_{t s}^{r, I}$, which ensures that the time 1 map $\mu_{t s}$ will be well-defined on some ball of fixed radius provided $t-s$ is small enough. So we need to set up a framework where approximate flows are replaced by some kind of local approximate flows, not defined in the whole of $E$. This is what section 4.1 is about.

We use in that section the notation $B_{R}$ for the ball $\{x \in E ;|x| \leqslant R\}$.

\subsection{Local approximate flows}

We provide in this section a version of the "approximate flow to flow" machinery that can be used to deal with rough differential equations involving inbounded vector fields with linear growth. It basically takes the same form as Theorem 2.1, with the uniform conditions replaced by well-chosen local controls.

Theorem 4.1. Let $c_{0}$ be a positive constant and $\mu$ be a continuous map from

$$
\bigcup_{x \in E}\{x\} \times\left\{(s, t) \in[0, T]^{2} ; 0 \leqslant t-s<c e^{-c_{0}|x|}\right\}
$$

to $E$, with $\mu_{t s}$ of class $\mathcal{C}^{1}$ on its domain, for any fixed $0 \leqslant s \leqslant t \leqslant T$. Assume this family of maps enjoys the following properties.

H1'. (Regularity)

(i) For all $R>0$ and $x \in B_{R}$, and for all $0 \leqslant s \leqslant t \leqslant T$ with $t-s \leqslant c e^{-c_{o} R}$,

(i $\left.\mathbf{i}_{\mathbf{1}}\right) \quad \mu_{t s}(x)=x+O_{c}\left(|t-s|^{1 /(2 p)}\right)$,

(i $\left.\mathbf{i}_{2}\right) \quad\left|D_{x} \mu_{t s}\right| \leqslant 1+O_{c}\left(|t-s|^{1 /(2 p)}\right)$.

for some positive constant $c$ independent of $R$.

(ii) There exists three positive constants a, $\rho, \alpha$, with $0<a(1-\rho)<\alpha<1$, such that one can write

$$
D_{x} \mu_{t s}=\mathrm{Id}+A_{x}^{t s}+B_{x}^{t s}
$$

for some $\mathrm{L}(E)$-valued $\rho$-Lipschitz maps $A^{\text {ts }}$ whose restrictions to every ball $B(R)$ has $\rho$-Lipschitz norm bounded above by $m_{R}|t-s|^{\alpha}$, for some constant $m_{R}$ depending only on $R$, and some maps $\mathrm{L}(E)$-valued maps $B^{\text {ts }}$, of class $\mathcal{C}^{1}$, whose restrictions to any ball $B(R)$ have $\mathcal{C}^{1}$-norm bounded above by $o_{m_{R}|t-s|^{1 / p}}(1)$. 
H2'. (Local $\mathcal{C}^{1}$-approximate flow property). There exists a positive constants $c_{1}$ such that the inequalities

$$
\begin{aligned}
\left|\mu_{t u}\left(\mu_{u s}(x)\right)-\mu_{t s}(x)\right| & \leqslant c e^{c_{1}|x|}|t-s|^{a} \\
\left|D_{x}\left(\mu_{t u} \circ \mu_{u s}\right)-D_{x} \mu_{t s}\right| & \leqslant c e^{c_{1}|x|}|t-s|^{a}
\end{aligned}
$$

hold for all $x \in B_{R}$, and all $0 \leqslant s \leqslant u \leqslant t \leqslant T$ with $\epsilon \leqslant \frac{u-s}{t-s} \leqslant 1-\epsilon$, and $t-s \leqslant \delta_{1}(R)$, for some function $\delta_{1}(R)$ no greater than $c e^{-c_{0} R}$.

Under these conditions, there exists a unique flow $\left(\varphi_{t s}\right)_{0 \leqslant s \leqslant t \leqslant T}$ on $E$ to which one can associate two functions $c, \varepsilon:(0, \infty) \rightarrow \mathbb{R}_{+}^{*}$, such that

$$
\left|\varphi_{t s}(x)-\mu_{t s}(x)\right| \leqslant c_{R}|t-s|^{a}
$$

holds for all $x \in B_{R}$, and all $0 \leqslant s \leqslant u \leqslant t \leqslant T$, with $t-s \leqslant \varepsilon(R)$, for all $R>0$.

A family of maps enjoying the properties listed in the above theorem is called a local $\mathcal{C}^{1}$-approximate flow with exponential growth. Note that given $0<\epsilon \leqslant 1 / 2$, it follows from $\mathbf{H 1}^{\prime}\left(\mathbf{i}_{\mathbf{1}}\right)$ that $\mu_{t u}\left(\mu_{u s}(x)\right)$ makes sense for $x \in B_{R}$ and any $0 \leqslant s \leqslant u \leqslant t \leqslant T$, with $t-s \leqslant c e^{-c_{o} R}$ and $\epsilon \leqslant \frac{u-s}{t-s} \leqslant 1-\epsilon$, provided $R$ is bigger than some radius $R_{\epsilon}$ depending only on $\epsilon$ and the data of the problem. The proof of Theorem 4.1 requires the following version of Lemma 2.3, where we set

$$
m_{\epsilon}=\max _{\epsilon \leqslant \beta \leqslant 1-\epsilon}\left\{\beta^{a}+(1-\beta)^{a}\right\}<1,
$$

and pick a constant $L$ strictly greater than

$$
\frac{2 c_{1}}{1-m_{\epsilon}} \vee\left(a-\frac{1}{2 p}\right) c_{0}
$$

Set $\delta_{1}(R)=c \exp \left(\frac{-1}{a}\left(L+\frac{c_{0}}{2 p}\right) R\right) \leqslant c e^{-c_{0} R}$. This function satisfies, for any $0 \leqslant$ $s \leqslant u \leqslant t \leqslant T$ with $t-s \leqslant \delta_{1}(R)$, and $\epsilon \leqslant \frac{u-s}{t-s} \leqslant 1-\epsilon$, the inequality

$$
\delta_{1}\left(R+c e^{-\frac{c_{0}}{2 p} R}\right) \geqslant(1-\epsilon) \delta_{1}(R) \geqslant t-u .
$$

Lemma 4.2. Let $\mu$ be a local $\mathcal{C}^{1}$-approximate flow with exponential growth. Given $\epsilon>0$, there exists a constant $R_{\epsilon}$ depending on the data of the problem such that for any $R \geqslant R_{\epsilon}$, any $0 \leqslant s \leqslant t \leqslant T$, with $t-s \leqslant \delta_{1}(R)$, and any $\epsilon$-special partition $\pi_{t s}$ of $(s, t)$, the map $\mu_{\pi_{t s}}(\cdot)$ is well-defined on $B_{R}$, and we have for any $x \in B_{R}$

$$
\left|\mu_{\pi_{t s}}(x)-\mu_{t s}(x)\right| \leqslant L e^{L|x|}|t-s|^{a} .
$$

Proof. Take $0 \leqslant s \leqslant t \leqslant T$, with $t-s \leqslant \delta_{1}(R)$. We proceed by induction on the number of subintervals of the partition $\pi_{t s}$, as in the proof of Lemma 2.3. With the notations adopted there, the induction hypothesis and assumption $\mathbf{H 1}{ }^{\prime}$ (i $\left.\mathbf{i}_{\mathbf{1}}\right)$ tell us that $\mu_{\pi_{u s}}(x)$ is well-defined and has norm bounded above by

$$
\left|\mu_{\pi_{u s}}(x)\right| \leqslant R+c|u-s|^{1 /(2 p)}+L e^{L R}|u-s|^{a} \leqslant R+c e^{-\frac{c_{0}}{2 p} R},
$$


so (4.4) ensures that $\mu_{t u}\left(\mu_{u s}(x)\right)$ is well-defined for $R$ big enough. For such radii, we have, from the decomposition given in (2.7),

$$
\begin{aligned}
\left|\mu_{\pi_{t s}}(x)-\mu_{t s}(x)\right| \leqslant & L e^{L\left|\mu_{\pi_{u s}(x)}\right|}|t-u|^{a}+\left(1+c e^{-\frac{c_{0} R}{2 p}}\right) L e^{L|x|}|u-s|^{a} \\
& +c_{1} e^{c_{1}|x|}|t-s|^{a}
\end{aligned}
$$

by using the induction hypothesis and assumptions H1' (i) to get the first two terms on the right hand side, and (4.1) to get the third term. So, denoting $o_{R}(1)=$ $c e^{-\frac{c_{0}}{2 p} R}$ and setting $u-s=\beta(t-s)$, with $\epsilon \leqslant \beta \leqslant 1-\epsilon$, we have the inequality

$$
\begin{aligned}
\left|\mu_{\pi_{t s}}(x)-\mu_{t s}(x)\right| & \leqslant\left\{\left(e^{o_{R}(1)}(1-\beta)^{a}+\left(\left(1+o_{R}(1)\right) \beta^{a}\right) L+c_{1}\right\} e^{L|x|}|t-s|^{a}\right. \\
& \leqslant\left(e^{o_{R}(1)} m_{\epsilon} L+c_{1}\right) e^{L|x|}|t-s|^{a} .
\end{aligned}
$$

One has $e^{o_{R}(1)} m_{\epsilon} L+c_{1} \leqslant L$, provided $R$ is bigger than some explicit radius $R_{\epsilon}$ depending only on $\epsilon$.

As in section 2 , one can get a $\mathcal{C}^{1}$ version of estimate (4.5), which takes here the following form.

Lemma 4.3. For every constant $c_{2}>c_{1}$, there exists some positive constant $R_{\epsilon}^{\prime}$, and a function $\delta:\left[R_{\epsilon}^{\prime}, \infty\right) \rightarrow \mathbb{R}_{+}$, such that

$$
\left|D_{x} \mu_{t s}^{n}-D_{x} \mu_{t s}\right| \leqslant c e^{c_{2} R}|t-s|^{a}
$$

holds for all $n \geqslant 2$ and all $x \in B_{R}$, whenever $t-s \leqslant \delta(R)$, and $R \geqslant R_{\epsilon}^{\prime}$.

Using this result, it is elementary to prove Theorem 4.1 using the same reasoning as in the proof of Theorem 2.1. We simply indicate the points that need to be amended, leaving the details to the reader.

Proof of theorem 4.1. a) Existence. Recall the definition of $\mu_{t s}^{(n)}$ given in (2.11), for dyadic times $0 \leqslant s \leqslant t \leqslant T$, ans write here $N_{n}$ for what we denoted by $N(n)$ in the proof of Theorem 2.1. Given $0 \leqslant i \leqslant N(n)-1$ and $1 \leqslant j \leqslant N(n)$, set

$$
\begin{aligned}
& f_{i}=\mu_{s_{i+1} s_{i}+2^{-n-1}} \circ \mu_{s_{i}+2^{-n-1} s_{i}}, \quad \text { and } \quad f_{[i]}=f_{i} \circ \cdots \circ f_{0}, \\
& g_{j}=\mu_{s_{j+1} s_{j}} \text { and } g^{[j]}=g_{N_{n}-1} \circ \cdots \circ g_{N_{n}-j} .
\end{aligned}
$$

Set $\varepsilon(R)=\delta(R) \wedge \delta_{1}(R)$, for all $R>0$, and take $s$ and $t$ such that $0 \leqslant t-s \leqslant \varepsilon(R)$. Then all the maps $f_{[i]}$ satisfy for all $x \in B_{R}$ the inequality

$$
\left|f_{[i]}(x)\right| \leqslant R+1 \text {. }
$$

Also, as the maps $g^{[j]}$ are of the form $\mu_{v u}^{m}$, for some $m \geqslant 1$ and $s \leqslant u \leqslant v \leqslant t$, there exists for any positive $R^{\prime}$ a constant $\kappa\left(R^{\prime}\right)$ such that the restrictions to $B_{R^{\prime}}$ of all the maps $g^{[j]}$ have Lipschitz constants uniformly bounded above by $\kappa\left(R^{\prime}\right)$, by Lemma 4.3. On the other hand, given $R>0$, it is a consequence of Lemma 4.2 
and assumption $\mathbf{H 1}$ ' that all the maps $f_{N_{n}-1-i} \circ f_{\left[N_{n}-2-i\right]}$ and $g_{N_{n}-1-i} \circ f_{\left[N_{n}-2-i\right]}$ send the ball $B_{R}$ into a fixed ball $B_{R^{\prime}}$. So, for $x \in B_{R}$, we have

$$
\begin{aligned}
\left|\mu_{t s}^{(n+1)}(x)-\mu_{t s}^{(n)}(x)\right| & \leqslant \kappa\left(R^{\prime}\right) \sum_{i=0}^{N_{n}-1}\left|\left(f_{N_{n}-1-i}-g_{N_{n}-1-i}\right)\left(f_{\left[N_{n}-2-i\right]}(x)\right)\right| \\
& \leqslant c_{1} \kappa\left(R^{\prime}\right) \sum_{i=0}^{N_{n}-1} e^{c_{1}\left|f_{\left[N_{n}-2-i\right]}(x)\right|}\left|s_{N_{n}-i}-s_{N_{n}-i-1}\right|^{a} \\
& \leqslant c_{1} \kappa\left(R^{\prime}\right) e^{(R+1) c_{1}} \sum_{i=0}^{N(n)-1}\left|s_{i+1}-s_{i}\right|^{a}
\end{aligned}
$$

the second inequality comes from assumption H2' and the third from (4.6). The existence of the flow $\varphi$ follows from that identity as in the proof of Theorem 2.1.

b) Uniqueness. One proceeds as in the proof of Theorem 2.1 by replacing the uniform estimate $\psi_{t s}=\mu_{t s}+O_{c}\left(|t-s|^{a}\right)$, by the local estimate $\psi_{v u}(x)=$ $\mu_{v u}(x)+O_{c}\left(|v-u|^{a}\right)$, which holds for all $s \leqslant u \leqslant v \leqslant t$ sufficiently close, all $|x| \leqslant R$ and an $R$-dependent constant $c$.

\subsection{Flows driven by weak geometric rough paths and vector fields with linear growth}

Let us go back to the setting and notations of section 3.2 and consider the ordinary differential equation

$$
\dot{y}_{u}=(t-s) V\left(y_{u}\right)+\sum_{r=1}^{[p]} \sum_{I \in \llbracket 1, \ell \rrbracket^{r}} \Lambda_{t s}^{r, I} V_{[I]}\left(y_{u}\right) .
$$

Assume the vector field $V$ is $(1+\rho)$-Lipschitz with linear growth, with $\rho>\frac{p-[p]}{p}$, and the $V_{i}$ are $\gamma$-Lipschitz with linear growth. Given $x \in E$, the maximal solution to equation (4.8) may well explode in finite time, due to the polynomial growth of the vector fields $V_{[I]}$. The function $\left|y_{u}\right|$ satisfies however an integral equation of the form

$$
\left|y_{u}\right| \leqslant|x|+a_{t s}+b_{t s} \int_{0}^{u}\left|y_{r}\right|^{[p]} d r
$$

with positive coefficients and $b_{t s} \leqslant c|t-s|^{1 / p}$, which guarantees that $y_{u}$ cannot explode before time $c /\left(|x|^{[p]-1}|t-s|^{1 / p}\right)$. So the time 1 map $\mu_{t s}$ is well-defined on the set

$$
\bigcup_{x \in E}\{x\} \times\left\{(s, t) \in[0, T]^{2} ; 0 \leqslant t-s<c e^{-c_{0}|x|}\right\},
$$

for a good choice of constants $c, c_{0}$. For $x \in B_{R}$ and $0 \leqslant t-s<c e^{-c_{0} R}$, one gets from the integral version of equation (4.8) the elementary estimate

$$
\left|y_{u}-x\right| \leqslant c\left(1+\|\mathbf{X}\|^{[p]}\right)(1+|x|) \exp \left(c\left(1+\|\mathbf{X}\|^{[p]}\right)|t-s|^{1 / p}\right)|t-s|^{1 / p} .
$$

As $R|t-s|^{1 /(2 p)} \leqslant c R e^{-c_{0} R /(2 p)} \leqslant c$, condition $\mathbf{H 1}{ }^{\prime}\left(\mathbf{i}_{\mathbf{1}}\right)$ holds as a consequence of the above estimate. 
Condition $\mathbf{H 1}$ ' $^{\left(\mathbf{i}_{2}\right)}$ holds for the same reason, and it follows from (4.9) and formula (3.16) that one has

$$
\left|\epsilon_{t s}^{f ;[p]}(x)\right| \leqslant c\left(1+\|\mathbf{X}\|^{\gamma}\right)\|f\|_{\gamma} e^{c\|\mathbf{X}\|^{[p]}|x|}|t-s|^{\gamma / p} .
$$

Similar estimates hold for the remainders $\epsilon_{t s}^{f ; n}$, for $1 \leqslant n \leqslant[p]-1$, and their first derivatives, with some exponent $a>1$ in place of $\gamma / p$, as in the proof of proposition 3.6. So it is straightforward to follow the proof of Proposition 3.6 and see that $\mu$ is a local $\mathcal{C}^{1}$-approximate flow for which one can choose for $c e^{c_{1}|x|}$ and $c e^{c_{3}|x|}$ in (4.1) and (4.2), respectively, an expression of the form $c\left(1+\|\mathbf{X}\|^{\gamma}\right)^{2} e^{c\|\mathbf{X}\|^{[p]}|x|}$. The decomposition of $\mu_{t s}$ given in (3.18) provides the definition of $A^{t s}$ and $B^{t s}$ used in checking that assumption H1' (ii) holds.

The flow $\left(\varphi_{t s}\right)_{0 \leqslant s \leqslant t \leqslant T}$ uniquely determined by $\mu$, by Theorem 4.1 , is called the solution flow to the rough differential equation

$$
d \varphi=V d t+\mathrm{F} \mathbf{X}(d t)
$$

on flows. It follows in particular from this well-posedness result that there is no explosion, which generalizes to our infinite dimensional setting the corresponding results of [18] and [17], which only treat the case $2 \leqslant p<3$ without a drift, and the finite dimensional analogue result of Friz-Victoir [16], who work with geometric rough paths.

\section{Paths driven by rough paths}

The results of section 3 provide an easy proof of sharp existence and well-posedness results for point dynamics driven by a Hölder weak geometric rough path. We adopt the notations of the preceeding sections.

Definition 5.1. Let $2<p<\gamma \leqslant[p]+1$ be given. Let $V_{1}, \ldots, V_{\ell}$ be vector fields on $E$ of class $\mathcal{C}^{[p]}$, and $\mathbf{X}$ be a Hölder weak geometric $p$-rough path on the time interval $[0, T]$. Let $V$ be a Lipschitz continuous vector field. A path $\left(z_{s} ; 0 \leqslant s \leqslant T\right)$ is said to solve the rough differential equation

$$
d z=V d t+F \mathbf{X}(d t)
$$

if there exists a constant $a>1$ independent of $\mathbf{X}$, and two possibly $\mathbf{X}$-dependent positive constants $\delta$ and $c$, such that

$$
\left|z_{t}-\mu_{t s}\left(z_{s}\right)\right| \leqslant c|t-s|^{a}
$$

holds for all $0 \leqslant s \leqslant t \leqslant T$, with $t-s \leqslant \delta$.

One can reformulate condition (5.2) under the form

$$
\left|z_{t}-\left\{z_{s}+(t-s) V\left(z_{s}\right)+\sum_{r=1}^{[p]} \sum_{I \in \llbracket 1, \ell \rrbracket^{r}} X_{t s}^{r, I} V_{I}\left(z_{s}\right)\right\}\right| \leqslant c|t-s|^{a}
$$

using Proposition 3.5. 
This form of the definition of a solution to a rough differential equation is due to Davie [9] for $2 \leqslant p<3$; it was extended to the general setting of Hölder geometric $p$ rough paths by Friz-Victoir [15] under a different form. We introduce the following compactness assumption in order to deal with some borderline existence case; it holds automatically in a finite dimensional setting.

(C) The vector fields $V_{1}, \ldots, V_{\ell}$ and $V_{[I]}$, with $I \in \llbracket 1, \ell \rrbracket^{r}$ and $r \leqslant[\gamma]$, map any ball $\left\{y \in E ;\left|y-y_{0}\right| \leqslant R\right\}$ into a compact set.

Theorem 5.2 (Existence/well-posedness).

a) Let $V$ be a bounded Lipschitz continuous vector field that maps balls into compact sets, and suppose that the $[\gamma]$-Lipschitz vector fields $V_{i}$ satisfy the compactness assumption (C). Then the rough differential equation (5.1) has a solution path.

b) This solution path is unique if the $V_{i}$ are $\gamma$-Lipschitz and $V$ is $(1+\rho)$ Lipschitz, for some $\rho>\frac{p-[p]}{p}$; it is in that case a continuous function of $\mathbf{X}$.

Note that we do not make any compactness assumption on $V$ or the $V_{i}$ in part $\mathbf{b}$ ) of the above statement. This part of Theorem 5.2 is usually called Lyons' universal limit theorem [19].

Proof. a) Existence. Given $\epsilon$ and $t \in[0, T]$, with $k \epsilon \leqslant t<(k+1) \epsilon$, set

$$
z_{t}^{\epsilon}=\left(\mu_{t k \epsilon} \circ \mu_{k \epsilon(k-1) \epsilon} \circ \cdots \circ \mu_{\epsilon 0}\right)\left(z_{0}\right) .
$$

We prove that the paths $\left(z_{t}^{\epsilon}\right)_{0 \leqslant t \leqslant T}$ have a uniformly converging subsequence by showing that they form an equicontinuous family and that the set

$$
\left\{\mu_{t}^{\epsilon}\right\}_{0<\epsilon \leqslant T}
$$

is precompact for any fixed $t \in[0, T]$.

First, for $k \epsilon \leqslant s<t \leqslant(k+1) \epsilon$, it is elementary to see that $z_{t}^{\epsilon}-z_{s}^{\epsilon}=$ $O_{c}\left\{|t-s|^{1 / p}\right\}$, for some constant $c$ independent of $\epsilon$. Now, given $0 \leqslant s<t \leqslant T$, with $k \epsilon \leqslant s<(k+1) \epsilon$, and $\ell \epsilon \leqslant t<(\ell+1) \epsilon$, with $k+1 \leqslant \ell$, one has

$$
z_{t}^{\epsilon}=\mu_{t \ell \epsilon} \circ\left(\bigcirc_{n=k+1}^{\ell-1} \mu_{(n+1) \epsilon n \epsilon}\right) \circ \mu_{(k+1) \epsilon s}\left(z_{s}^{\epsilon}\right) .
$$

As every partition of any interval into subintervals of equal length is of special type $1 / 2$, one has by Lemma 2.3 and Proposition 3.4,

$$
\bigcirc_{n=k+1}^{\ell-1} \mu_{(n+1) \epsilon n \epsilon}=\mu_{\ell \epsilon(k+1) \epsilon}+O_{L}\left(|(k-\ell) \epsilon|^{\gamma / p}\right)
$$

provided $t-s \leqslant \delta$. Since the maps $\mu_{a b}$ are uniformly Lipschitz continuous, the approximate flow property of $\mu$ gives

$$
z_{t}^{\epsilon}=\mu_{t s}\left(z_{s}^{\epsilon}\right)+O_{L+c}\left(|t-s|^{\frac{\gamma}{p}}\right) .
$$

The equicontinuity of the family of paths $\left(z_{\bullet}^{\epsilon}\right)_{0<\epsilon \leqslant 1}$ follows from the above inequality and the fact that $\left\|\mu_{t s}-\mathrm{Id}\right\|_{\infty} \leqslant c|t-s|^{1 / p}$. 
Fix $t \in[0, T]$. To see that the set $\left\{\mu_{t}^{\epsilon}\right\}_{0<\epsilon \leqslant T}$ is pre-compact, note first that for any open ball $B$ of $E$, the set $\left\{\mu_{b a}(z)-z ; z \in B, 0 \leqslant a \leqslant b \leqslant T\right\}$ is compact. Indeed, as

$$
\mu_{b a}(z)-z=(b-a) \int_{0}^{1} V\left(y_{u}\right) d u+\Lambda_{b a}^{I} \int_{0}^{1} V_{[I]}\left(y_{u}\right) d u
$$

and $\left(y_{u}\right)_{0 \leqslant u \leqslant 1}$ remains in a fixed ball for $y_{0}$ ranging in $B$, the above integrals belong to a fixed compact convex set independent of $a$ and $b$, by the compactness assumption on $V$ and assumption (C) on the $V_{i}$, and a well-known theorem of Mazur on convex hulls of compact subsets of Banach spaces. It follows from this fact and (5.3) that we have for any $N \geqslant 1$ and $s_{i}=i t / N$,

$$
\begin{aligned}
z_{t}^{\epsilon}-z_{0} & =\sum_{i=0}^{N-1}\left\{\left\{\mu_{s_{i+1} s_{i}}\left(z_{s_{i}}^{\epsilon}\right)-z_{s_{i}}^{\epsilon}\right\}+O_{L+c}(t / N)^{\gamma / p}\right\} \\
& =o_{N}(1)+\sum_{i=0}^{N-1}\left\{\mu_{s_{i+1} s_{i}}\left(z_{s_{i}}^{\epsilon}\right)-z_{s_{i}}^{\epsilon}\right\} .
\end{aligned}
$$

So there exists a compact set $K(N)$ depending only on $N$ such that

$$
\left\{\mu_{t}^{\epsilon}\right\}_{0<\epsilon \leqslant T} \subset\left\{K(N)+o_{N}(1)\right\},
$$

which implies the pre-compactness of the set $\left\{\mu_{t}^{\epsilon}\right\}_{0<\epsilon \leqslant T}$.

Ascoli's theorem ensures as a consequence the pre-compactness of the set of paths $\left\{\mu_{\bullet}^{\epsilon}\right\}_{0<\epsilon \leqslant T}$ in the uniform topology. We see that any limit path satisfies (5.2) for all $0 \leqslant s \leqslant t \leqslant T$ with $t-s \leqslant \delta$, by sending $\epsilon$ to 0 .

b) Uniqueness. Set $a=\min (1+\rho / p, \gamma / p)$. To prove uniqueness of the solution when the $V_{i}$ are $\gamma$-Lipschitz and $V$ is $(1+\rho)$-Lipschitz, note first that $z_{\bullet}:=\varphi_{\bullet}\left(z_{0}\right)$ is a solution. Let $y_{\bullet}$ be any other solution. From the fact that the maps $\varphi_{t s}$ are uniformly Lipschitz continuous, with a Lipschitz constant bounded above by $L$ say, one can write for any $\epsilon>0$ and any integer $k \leqslant T / \epsilon$,

$$
\begin{aligned}
y_{k \epsilon} & =\varphi_{k \epsilon,(k-1) \epsilon}\left(y_{(k-1) \epsilon}\right)+O_{c}\left(\epsilon^{a}\right) \\
& =\varphi_{k \epsilon,(k-1) \epsilon}\left(\varphi_{(k-1) \epsilon,(k-2) \epsilon}\left(y_{(k-2) \epsilon}\right)+O_{c}\left(\epsilon^{a}\right)\right)+O_{c}\left(\epsilon^{a}\right) \\
& =\varphi_{k \epsilon,(k-2) \epsilon}\left(y_{(k-2) \epsilon}\right)+O_{c L}\left(\epsilon^{a}\right)+O_{c}\left(\epsilon^{a}\right),
\end{aligned}
$$

and see by induction that

$$
\begin{aligned}
y_{k \epsilon} & =\varphi_{k \epsilon,(k-n) \epsilon}\left(y_{(k-n) \epsilon}\right)+O_{c L}\left((n-1) \epsilon^{a}\right)+O_{c}\left(\epsilon^{a}\right) \\
& =\varphi_{k \epsilon, 0}(x)+O_{c L}\left(k \epsilon^{a}\right)+o_{\epsilon}(1)=z_{k \epsilon}+O_{c L}\left(k \epsilon^{a}\right)+o_{\epsilon}(1) .
\end{aligned}
$$

Taking $\epsilon$ and $k$ so that $k \epsilon$ converges to some $t \in[0, T]$, we see that $y_{t}=z_{t}$, since $a>1$.

The continuous dependence of the solution path $z_{\bullet}$ with respect to $\mathbf{X}$ is transferred from $\varphi$ to $z_{\bullet}$. 
Remark 5.3. The existence statement a) was first proved in a finite dimensional setting by Davie [9]. Caruana [5] obtained an existence result in a Banach space setting, working with a full rough differential equation under a similar compactness assumption as (C), but for a rough differential equation with no drift term. He is able, on the other side, to deal with the weaker and optimal regularity assumption that the $V_{i}$ be $(\gamma-1)$-Lipschitz. However, as full rough differential equations enter the framework of rough differential equations driven by vector fields with linear growth, one recovers and extends in a simpler and (much) shorter way the main content of Caruana's result using Lemma 4.2 instead of Lemma 2.3 in the above existence proof.

\section{An illustration: mean field stochastic rough differential equations}

We show in this section how the results of sections 3 can be used to study some simple mean field stochastic rough differential equations. This kind of dynamics pops in naturally in the study of the large population limit of some classes of interacting random evolutions. The interaction holds through the dependence of the local characteristics of the random motion of each particle on the empirical measure of the whole family of particles. In a diffusion setting, each particle $i$ would satisfy a stochastic differential equation of the form

$$
d x_{t}^{(i)}=b\left(x_{t}^{(i)}, \mu_{t}^{N}\right) d t+\sigma\left(x_{t}^{(i)}, \mu_{t}^{N}\right) d B_{t}^{(i)},
$$

where $\mu_{t}^{N}=\frac{1}{N} \sum_{k=1}^{N} \delta_{x_{t}^{(k)}}$. A large industry has been devoted to showing that the limit distribution in paths space of a typical particle of the system when $N$ tends to infinity has a dynamics of the form

$$
d x_{t}=b\left(x_{t}, \mathcal{L}\left(x_{t}\right)\right) d t+\sigma\left(x_{t}, \mathcal{L}\left(x_{t}\right)\right) d B_{t},
$$

where $\mathcal{L}\left(x_{t}\right)$ stands for the law of $x_{t}$. Theorem 6.2 provides a well-posedness result for such a limit equation, in the context of rough differential equations. As emphasized in [6], almost all the works in this area are set in the framework of a filtered probability space and rely crucially on some martingale arguments. On the other hand, the increasing importance of non-semi-martingale processes, like fractional Brownian motion, makes it desirable to have some more flexible tools to investigate equation (6.1) in such contexts. The theory of rough paths developed above provides a nice framework for that.

A few notations are needed to set the problem. Given $2 \leqslant p<\gamma \leqslant[p]+1$, we equip the set $\mathcal{M}_{1}\left(\mathbb{R}^{d}\right)$ of probability measures on $\mathbb{R}^{d}$ with the metric induced by its embedding in the dual of $\mathcal{C}^{\gamma}\left(\mathbb{R}^{d}\right)$ :

$$
\mathrm{d}(\mu, \nu)=\sup \left\{(g, \mu)-(g, \nu) ; g \in \mathcal{C}^{\gamma}\left(\mathbb{R}^{d}\right),\|g\|_{\gamma} \leqslant 1\right\} .
$$

This metric topology is stronger than the weak convergence topology. Given any positive constant $m$, note that the set $\operatorname{Lip}(m)$ of Lipschitz continuous paths from $[0, T]$ to $\left(\mathcal{M}_{1}\left(\mathbb{R}^{d}\right), \mathrm{d}\right)$, with Lipschitz constant no greater than $m$, is closed under the norm of uniform convergence, so it is a Banach space. 
Fix $T>0$ and suppose $\mathbf{X}$ is a random variable defined on some probability space $(\Omega, \mathcal{F}, \mathbb{P})$, with values in the set of Hölder weak geometric $p$-rough paths over $\mathbb{R}^{\ell}$, on the time interval $[0, T]$; write $\mathbf{X}=1 \oplus X^{1} \oplus \cdots \oplus X^{[p]}$, and $\left(\mathcal{F}_{t}\right)_{0 \leqslant t \leqslant T}$ for the filtration generated by $\mathbf{X}$, with $\mathcal{F}_{t}=\sigma\left(\mathbf{X}_{v u} ; 0 \leqslant u \leqslant v \leqslant t\right)$.

Let $V, V_{1}, \ldots, V_{\ell}: \mathbb{R}^{d} \times \mathcal{M}_{1}\left(\mathbb{R}^{d}\right) \rightarrow \mathbb{R}^{d}$ be measure-dependent vector fields on $\mathbb{R}^{d}$, and $\mathrm{F}$ stands for a collection $\left(V_{1}, \ldots, V_{\ell}\right)$. Given a Lipschitz continuous path $\mathcal{P}=\left(P_{t}\right)_{0 \leqslant t \leqslant T}$ in $\left(\mathcal{M}_{1}\left(\mathbb{R}^{d}\right), \mathrm{d}\right)$ and $\omega \in \Omega$, denote by $x_{\bullet}(\omega)$ the unique solution (under appropriate conditions) to the rough differential equation on paths

$$
d x_{t}=V\left(x_{t}, P_{t}\right) d t+\mathrm{F}\left(x_{t}, P_{t}\right) \mathbf{X}(d t)
$$

where $x_{0}$ may be an integrable random variable independent of $\mathbf{X}$. Denote by $\Phi(\mathcal{P})_{t}$ the law of $x_{t}$.

Definition 6.1. A solution $x_{\bullet}$ to (6.2) for which

$$
\Phi(\mathcal{P})_{t}=P_{t}
$$

for all $0 \leqslant t \leqslant T$, is said to be a solution of the nonlinear rough differential equation (or mean field stochastic rough differential equation)

$$
d x_{t}=V\left(x_{t}, \mathcal{L}\left(x_{t}\right)\right) d t+\mathrm{F}\left(x_{t}, \mathcal{L}\left(x_{t}\right)\right) \mathbf{X}(d t) .
$$

Theorem 6.2 below provides conditions on the vector fields $V, \mathrm{~F}$ and the rough path $\mathbf{X}$ under which existence and uniqueness for solutions of this equation can be proved.

Theorem 6.2. Given any $P \in \mathcal{M}_{1}\left(\mathbb{R}^{d}\right)$, we assume that $V(\cdot, P)$ is of class $\mathcal{C}^{2+[p]}$, with associated norm no greater than $\lambda$, and that the vector fields $V_{i}(\cdot, P)$ are of class $\mathcal{C}^{2[p]+1}$, with associated norms uniformly bounded with respect to $P$, and that they satisfy the inequalities

$$
\max _{i=1, \ldots, \ell}\left\|V_{i}(\cdot, P)-V_{i}(\cdot, Q)\right\|_{\infty} \vee\|V(\cdot, P)-V(\cdot, Q)\|_{\infty} \leqslant \lambda \mathrm{d}(P, Q),
$$

for all $P, Q \in \mathcal{M}_{1}\left(\mathbb{R}^{d}\right)$.

(1) Assume the vector fields $V_{i}(\cdot, P)=V_{i}(\cdot)$ do not depend on their $\mathcal{M}_{1}\left(\mathbb{R}^{d}\right)$ component, and the polynomial moment condition

$$
\mathbb{E}\left[\|\mathbf{X}\|^{([p]+1)^{2}}\right]<\infty .
$$

Then the map $\Phi$ has a unique fixed point in $\operatorname{Lip}(m)$, for any positive constant $m$; it depends continuously on the law of $\mathbf{X}$.

(2) In the general case where the vector fields $V_{i}(\cdot, P)$ are allowed to depend on their $\mathcal{M}_{1}\left(\mathbb{R}^{d}\right)$-component, assume that the random variables

$$
\left(X_{b a}^{r, I}\right)_{r=1, \ldots,[p], I \in \llbracket 1, \ell \rrbracket^{r}}
$$


are integrable and that there exists for each $0 \leqslant a \leqslant T$, a positive random variable $C_{a}$, such that each of them satisfies the inequality

$$
\left|\mathbb{E}\left[X_{b a}^{r, I} \mid \mathcal{F}_{a}\right]\right| \leqslant C_{a}(b-a),
$$

for all $0 \leqslant a \leqslant b \leqslant T$, with $\sup _{0 \leqslant a \leqslant T} \mathbb{E}\left[C_{a}\right]<\infty$. Then one can choose $m$ big enough so that the map $\Phi$ has a fixed point in $\operatorname{Lip}(m)$.

Assumption (6.5) holds for instance for the Brownian rough path, the rough path above an Orsntein-Uhlenbeck process, or fractional Brownian motion with Hurst index no smaller than $1 / 2$. The above regularity assumptions on $V$ and the $V_{i}$ ensure that the solution flow to the rough differential equation (6.2) is of class $\mathcal{C}^{[p]}$, with polynomial bounds on the size of its derivatives, in terms of $\|\mathbf{X}\|$. The moment condition (6.4) ensures the integrability of all these bounds.

Proof. Fix $P_{0} \in \mathcal{M}_{1}\left(\mathbb{R}^{d}\right)$ and define $\operatorname{Lip}_{0}(m)$ as the subset of $\operatorname{Lip}(m)$ paths with starting point $P_{0}$.

(1) Uniqueness. We suppose in this paragraph that the vector fields $V_{i}$ do not depend on their $\mathcal{M}_{1}\left(\mathbb{R}^{d}\right)$-component. First, we prove that $\Phi$ is a strict contraction of $\operatorname{Lip}_{0}(m)$, provided $T$ is small enough. We use for that purpose a decomposition which is reminiscent of a well-known trick in the setting of Markov processes used to compare two different semigroups. Indeed, given two semigroups $\left(\mathrm{T}_{t}\right)_{t \geqslant 0}$ and $\left(\mathrm{T}_{t}^{\prime}\right)_{t \geqslant 0}$, with generators $A$ and $A^{\prime}$ respectively, the identity

$$
T_{t}-T_{t}^{\prime}=\int_{0}^{t}\left\{A\left(T_{r}-T_{r}^{\prime}\right)+\left(A-A^{\prime}\right) T_{r}\right\} d r
$$

provides a direct comparison of $T_{t}$ and $T_{t}^{\prime}$.

Fix $\omega \in \Omega$ and omit it in the notations of this paragraph. Denote by $\mu_{t s}^{\mathcal{Q}}$ the approximate flow associated to the time non-homogeneous rough differential equation (6.2), with $Q_{t}$ instead of $P_{t}$, for all $0 \leqslant t \leqslant T$. Given $g \in \mathcal{C}^{\gamma}\left(\mathbb{R}^{d}\right)$, and setting $s_{i}=s+i 2^{-n}(t-s)$, we have

$$
\begin{array}{rl}
\mathbb{E}\left[g\left(\varphi_{t 0}^{\mathcal{P}}\left(x_{0}\right)\right)-g\left(\varphi_{t 0}^{\mathcal{Q}}\left(x_{0}\right)\right)\right]=\sum_{k=0}^{2^{n}-1} & \mathbb{E}\left[\left\{g\left(\varphi_{s_{2}{ }^{2} s_{2^{n}-k}}^{\mathcal{Q}} \circ \varphi_{s_{2^{n}-k} s_{2^{n}-k-1}}^{\mathcal{P}}\right)\right.\right. \\
& \left.\left.-g\left(\varphi_{s_{2} s_{2^{n}-k}}^{\mathcal{Q}} \circ \varphi_{s_{2^{n}-k} s_{2^{n}-k-1}}^{\mathcal{Q}}\right)\right\} \circ \varphi_{s_{2^{n}-k-1} s_{0}}^{\mathcal{P}}\left(x_{0}\right)\right],
\end{array}
$$

with the obvious convention concerning the summand for the first and last term of the sum. It follows from the time-inhomogeneous version of Proposition 3.5 giving

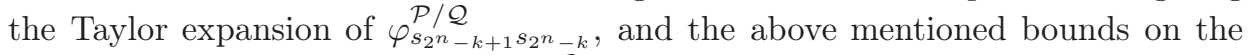
size of the derivatives of the maps $\varphi_{b a}^{\mathcal{Q}}$, for $0 \leqslant a \leqslant b \leqslant T$, that

$$
\left|\mathbb{E}\left[g\left(\varphi_{t 0}^{\mathcal{P}}\left(x_{0}\right)\right)-g\left(\varphi_{t 0}^{\mathcal{Q}}\left(x_{0}\right)\right)\right]\right|
$$

is no greater than the large $n$ limit of

$$
\begin{aligned}
& \mathbb{E}\left[c\left(1+\|\mathbf{X}\|^{\gamma}\right)\|g\|_{\mathcal{C}^{1}} \sum_{k=0}^{2^{n}-1}\left\|V\left(\cdot, P_{s^{n}-k-1}\right)-V\left(\cdot, Q_{s_{2^{n}-k-1}}\right)\right\|_{\infty} 2^{-n} t\right] \\
& +c\|g\|_{\gamma} \mathbb{E}\left[1+\|\mathbf{X}\|^{([p]+1)^{2}}\right] 2^{\frac{\gamma-p}{p} n} .
\end{aligned}
$$


This upper bound is no greater than

$$
c\|g\|_{\mathcal{C}^{\gamma}} \lambda 2^{-n} t \sum_{k=0}^{2^{n}-1} \mathrm{~d}\left(P_{s_{n-k-1}}, Q_{s_{n-k-1}}\right)+o_{n}(1)
$$

that is

$$
c t\|g\|_{\mathcal{C}^{\gamma}} \sup _{s \in[0, t]} \mathrm{d}\left(P_{s}, Q_{s}\right)+o_{n}(1)
$$

since $\mathbb{E}\left[\|\mathbf{X}\|^{([p]+1)^{2}}\right]$ is finite. As a result, we have for all $t \in[0, T]$,

$$
\mathrm{d}\left(\Phi(\mathcal{P})_{t}, \Phi(\mathcal{Q})_{t}\right)=\sup _{\|g\|_{\mathcal{C} \gamma} \leqslant 1}\left|\mathbb{E}\left[g\left(\varphi_{t 0}^{\mathcal{P}}\left(\mathbf{x}_{0}\right)\right)\right]-\mathbb{E}\left[g\left(\varphi_{t 0}^{\mathcal{Q}}\left(\mathbf{x}_{0}\right)\right)\right]\right| \leqslant c T \max _{t \in[0, T]} \mathrm{d}\left(P_{t}, Q_{t}\right)
$$

so $\Phi$ is a strict contraction provided $c T<1$.

As usual in the study of ordinary differential equations, the fact that $\Phi$ has a unique fixed point for any $T$ follows from the fact that the above condition on $T$ does not involve $P_{0}=Q_{0}$.

As the solution to the rough differential equation (6.2) depends continuously on $\mathbf{X}$, the map $\Phi$, considered as a function of $\mathcal{P}$ and of the parameter "law of $\mathbf{X}$ ", is a continuous function of its two arguments. It is an elementary result that the unique fixed point of $\Phi$ is then a continuous function of the parameter.

(2) Existence. We first prove that one can choose $m$ big enough so that the map $\Phi$ sends $\operatorname{Lip}(m)$ into itself. Given an $m$-Lipschitz continuous path $\mathcal{P}$, the time-dependent bounded vector field $V\left(x, P_{t}\right)$ is Lipschitz in its two arguments, so we can denote by $\varphi^{\mathcal{P}}$ the well-defined solution flow to the time non-homogeneous rough differential equation $(6.2)$ on $\mathbb{R}^{d}$. We need to see that, for any function $g \in \mathcal{C}^{\gamma}\left(\mathbb{R}^{d}\right)$, with $\|g\|_{\gamma} \leqslant 1$, and any $0 \leqslant s \leqslant t \leqslant T$, we have

$$
\left(g, \Phi(\mathcal{P})_{t}\right)-\left(g, \Phi(\mathcal{P})_{s}\right) \leqslant c(t-s),
$$

for some positive constant $c \leqslant m$, that is

$$
\mathbb{E}\left[g\left(\varphi_{t 0}^{\mathcal{P}}\left(x_{0}\right)\right)-g\left(\varphi_{s 0}^{\mathcal{P}}\left(x_{0}\right)\right)\right] \leqslant c(t-s)
$$

Write $\varphi_{t s}$ for $\varphi_{t s}^{\mathcal{P}}$, as $\mathcal{P}$ is fixed in this paragraph; write also $z$ for $\varphi_{s 0}\left(x_{0}\right)$. Then, the telescopic decomposition

$$
\begin{aligned}
(\star) & :=\mathbb{E}\left[g\left(\varphi_{t 0}\left(x_{0}\right)\right)-g\left(\varphi_{s 0}\left(x_{0}\right)\right)\right]=\mathbb{E}\left[g\left(\varphi_{t s}(z)\right)-g(z)\right] \\
& =\sum_{k=0}^{2^{n}-1} \mathbb{E}\left[g\left(\varphi_{s_{k+1} s_{k}}\left(\varphi_{s_{k} s_{0}}(z)\right)\right)-g\left(\varphi_{s_{k} s_{0}}(z)\right)\right],
\end{aligned}
$$

together with the uniform Euler-Taylor expansion for $\varphi_{s_{k+1} s_{k}}$ given in (3.15) and (3.20), and the Lebesgue dominated convergence theorem, show that $(\star)$ is 
no greater than the large $n$ limit of

$$
\begin{aligned}
& \sum_{k=0}^{2^{n}-1} \mathbb{E}\left[2^{-n}(t-s)\left(V\left(\cdot, P_{s_{k}}\right) g\right)\left(\varphi_{s_{k} s_{0}}(z)\right)\right. \\
& \quad+\sum_{r=1}^{[p]} \sum_{I \in \llbracket 1, \ell \rrbracket^{r}} X_{s_{k+1} s_{k}}^{r, I}\left(V_{I}\left(\cdot, P_{s_{k}}\right) g\right)\left(\varphi_{s_{k} s_{0}}(z)\right)+\|g\|_{\gamma}\left(2+\|\mathbf{X}\|^{\gamma}\right) o\left(\left(2^{-\frac{\gamma}{p} n}\right)\right],
\end{aligned}
$$

where the term $o(\cdot)$ above does not depend on the rough path $\mathbf{X}$. So, using the conditions (6.4) and (6.5) of the theorem, and the uniform boundedness assumption in $\mathcal{C}^{[p]+1}$ of the vector fields $V_{i}(\cdot, P)$, the quantity $(\star)$ appears as no greater than the large $n$ limit of

$$
\begin{aligned}
& c(t-s)+\sum_{k=0}^{2^{n}-1} \sum_{r=1}^{[p]} \sum_{I \in \llbracket 1, \ell \rrbracket^{r}} \mathbb{E}\left[\mathbb{E}\left[X_{s_{k+1} s_{k}}^{r, I} \mid \mathcal{F}_{s_{k}}\right]\left(V_{I}\left(\cdot, P_{s_{k}}\right) g\right)\left(\varphi_{s_{k} s_{0}}(z)\right)\right]+o_{n}(1) \\
& \leqslant c(t-s)+c \sum_{k=0}^{2^{n}-1} \mathbb{E}\left[C_{s_{k}}\right]\left(s_{k+1}-s_{k}\right)+o_{n}(1) \leqslant c(t-s)+o_{n}(1) .
\end{aligned}
$$

Inequality (6.6) follows by choosing $m$ big enough. As $\operatorname{Lip}_{0}(m)$ is a convex compact subset of the set of continuous paths from $[0, T]$ to $\left(\mathcal{M}_{1}\left(\mathbb{R}^{d}\right), \mathrm{d}\right)$, equipped with the norm of uniform convergence, Schauder's fixed point theorem applies and gives the existence of a fixed point of the map $\Phi$.

Remark 6.3. So far there has been only one other work dealing with mean field stochastic rough differential equations, namely Cass and Lyons [6]. They prove a well-posedness result under more restrictive assumptions on the vector fields and the driving rough path, asking for a linear mean field interaction in the drift and no mean field interaction in the $V_{i}$ 's, and requiring exponential moments for the accumulated local variation of $\mathbf{X}$. They have no existence result. As they work with Wassersein distance on $\mathcal{M}_{1}\left(\mathbb{R}^{d}\right)$, our results and theirs are not directly comparable, but the assumptions of Theorem 6.2 are significantly weaker when both settings apply. This covers in particular the case of Gaussian $p$-rough paths, with $2 \leqslant p<4$.

\section{References}

[1] Azencott, R.: Formule de Taylor stochastique et développement asymptotique d'intégrales de Feynman. In Séminaire de probabilité XVI, Supplément, 237-285. Lecture Notes in Mathematics 921, Springer, Berlin-New York, 1982.

[2] Bailleul, I.: Flows driven by Banach space-valued rough paths. In Séminaire Probabilités XLVI, 195-205. Lecture Notes in Mathematics 2123, Springer, Heidelberg, 2014.

[3] Bailleul, I. and Riedel, S.: Rough flows. Preprint, arXiv: 1505.01692, 2015. 
[4] Ben Arous, G.: Flots et séries de Taylor stochastiques. Probab. Theory Related Fields 81 (1989), no. 1, 29-77.

[5] Caruana, M.: Peano's theorem for rough differential equations in infinitedimensional Banach spaces. Proc. London Math. Soc. (3) 100 (2010), no. 1, 177-215.

[6] Cass, T. and Lyons, T.: Evolving communities with indiviual preference. Proc. London Math. Soc. 110 (2015), no. 1, 83-107.

[7] Castell, F.: Asymptotic expansion of stochastic flows. Probab. Theory Related Fields 96 (1993), no. 2, 225-239.

[8] Chen, K. T.: Iterated path integrals. Bull. Amer. Math. Soc. 83 (1977), no. 5, 831-879.

[9] Davie, A. M.: Differential equations driven by rough paths: an approach via discrete approximation. Appl. Math. Res. Express. AMRX 2007, no. 2, 40 pp.

[10] Feyel, D. and De La Pradelle, A.: Curvilinear integrals along enriched paths. Electron. J. Probab. 11 (2006), 860-892.

[11] Feyel, D., De la Pradelle, A. and Mokobodzki, G.: A non-commutative sewing lemma. Electron. Commun. Probab. 13 (2008), 24-34.

[12] Friz, P. And Hairer, M.: A course on rough paths. With an introduction to regularty structures. Universitext, Springer, Cham, 2014.

[13] Friz, P. and Oberhauser, H.: Rough paths limits of the Wong-Zakai type with a modified drift term. J. Func. Anal. 256 (2009), 3236-3256.

[14] Friz, P. And Victoir, N.: A note on the notion of geometric rough paths. Probab. Theory Related Fields 136 (2006), no. 3, 395-416.

[15] Friz, P. And Victoir, N.: Euler estimates for rough differential equations. J. Differential Equations 244 (2008), no. 2, 388-412.

[16] Friz, P. And Victoir, N.: Multidimensional stochastic processes as rough paths. Cambridge Studies in Advanced Mathematics 120, Cambridge University Press, Cambridge, 2010.

[17] Lejay, A.: On rough differential equations. Electron. J. Probab. 14 (2009), 341-364

[18] Lejay, A.: Global solutions to rough differential equations with unbounded vector fields. In Séminaire de probabilités XLIV, 215-246. Lecture Notes in Mathematics 2046, Springer, Heidelberg, 2012.

[19] Lyons, T.: Differential equations driven by rough signals. Rev. Mat. Iberoamericana 14 (1998), no. 2, 215-310

[20] Lyons, T. J. and Caruana, M. and Lévy, Th.: Differential equations driven by rough paths. Lecture Notes in Mathematics 1908, Springer, Berlin, 2007.

[21] Lyons, T. And Qian, Z.: System control and rough paths. Oxford Mathematical Monographs, Oxford University Press, Oxford, 2002.

[22] Strichartz, R.: The Campbell-Baker-Hausdorff-Dynkin formula and solutions of differential equations. J. Funct. Anal. 72 (1987), 320-345.

Received September 6, 2013.

Ismaël Bailleul: IRMAR, 263 Avenue du General Leclerc, 35042 Rennes, France.

E-mail: ismael.bailleul@univ-rennes1.fr 